\title{
FILTERED MODULES WITH COEFFICIENTS
}

\author{
EKNATH GHATE AND ARIANE MÉZARD
}

\begin{abstract}
We classify the filtered modules with coefficients corresponding to two-dimensional potentially semi-stable $p$-adic representations of the Galois group of $\mathbf{Q}_{p}$ under some assumptions (e.g., $p$ is odd). We focus on the new features that arise when the coefficients are not necessarily $\mathbf{Q}_{p}$
\end{abstract}

\section{INTRODUCTION}

Let $E$ be a finite extension of $\mathbf{Q}_{p}$ and let $\rho: \operatorname{Gal}\left(\overline{\mathbf{Q}}_{p} / \mathbf{Q}_{p}\right) \rightarrow \mathrm{GL}_{2}(E)$ be a continuous twodimensional local $p$-adic Galois representation with coefficients in $E$. In recent work towards a p-adic local Langlands correspondence, Breuil has suggested associating to a potentially semi-stable representation $\rho$ a $\mathrm{GL}_{2}\left(\mathbf{Q}_{p}\right)$-Banach space $\Pi(\rho)$ which determines $\rho$ up to isomorphism [Bre03], [Bre04].

Following the work of Breuil, a new technique was introduced for semi-stable (non-crystalline) representations by Colmez [Col04] using the theory of $(\varphi, \Gamma)$-modules. Using this technique, Berger and Breuil [BB06] have treated the case where $\rho$ is 'crystabeline', that is, $\rho$ becomes crystalline over a potentially abelian extension of $\mathbf{Q}_{p}$. The case where $\rho$ is 'trianguline' has been treated by Colmez in [Col05], [Col07]. Special cases have also been proved by Breuil and Mézard [BM05]. The correspondence $\rho \mapsto \Pi(\rho)$ is expected to be compatible with similar modulo $p$ correspondences ([BM05], [Ber05]). Also the representations $\Pi(\rho)$ appear in the completed étale cohomology of $p$-towers of modular curves (see the work of Breuil and Emerton [Bre04], [Eme05], [BE05]).

After the fundamental theorem of Colmez and Fontaine [CF00], potentially semi-stable Galois representations $\rho$ as above are described by admissible filtered $(\varphi, N)$-modules with coefficients. A very first step in the construction of the $p$-adic local Langlands correspondence is to write down these filtered modules. An independent but closely related question is to identify those filtered modules with coefficients arising from the local components of Galois representations attached to elliptic modular eigenforms.

The goal of this paper is to write down new admissible filtered $(\varphi, N)$-modules with coefficients $E$ corresponding to two-dimensional representations $\rho$. The emphasis is on understanding what new features arise when $E$ is a general finite extension of $\mathbf{Q}_{p}$. Recall that the case $E=\mathbf{Q}_{p}$ was treated completely by Fontaine and Mazur [FM95, app. A] at least if $p \geq 5$ (see also Volkov [Vol01]). The study of the case where $E$ is arbitrary was initiated by Breuil and Mézard [BM02]. More recently

Date: May 2008. 
Savitt [Sav05] has treated cases where $\rho$ becomes crystalline over a tamely ramified extension of $\mathbf{Q}_{p}$. In this paper we will extend some of the results in these papers. In particular we will treat cases where $\rho$ becomes crystalline over wildly ramified extensions of $\mathbf{Q}_{p}$. A novel feature of our work is that we write down explicit Galois stable lines which are candidates for the filtration. Computing these lines turns out to be more than just an exercise, since in general one is no longer working in a vector space over a field but in an $F \otimes_{F_{0}} E$-module, where $F$ is a finite, often wildly ramified, extension of $\mathbf{Q}_{p}$, and $F_{0}$ is the maximal unramified extension of $\mathbf{Q}_{p}$ in $F$.

The text is organized as follows: the first part is devoted to definitions and notation concerning filtered modules with coefficients $(\S 2.1)$, potentially semi-stable representations ( $(2.3)$ and Galois types (§2.5). Roughly, the Galois type of a potentially semi-stable representation $\rho$ is the WeilDeligne representation $\left.W D(\rho)\right|_{I_{p}}$ restricted to the inertia group of $\operatorname{Gal}\left(\overline{\mathbf{Q}}_{p} / \mathbf{Q}_{p}\right)$. There is nothing original in this section, but for the sake of completeness we present some of the tools we will need for our study. The computations of the filtered modules are carried out in the second part. We consider four different cases depending on the Galois type of $\rho$ : special case ( $\S 3.1)$, ramified principal series cases (§3.2), unramified supercuspidal case (§3.3) and ramified supercuspidal case (§3.4).

\section{Preliminaries}

2.1. Filtered modules with coefficients. Let $p$ be a prime. Let $F$ be a finite Galois extension of $\mathbf{Q}_{p}$ and let $F_{0}$ be the maximal unramified extension of $\mathbf{Q}_{p}$ contained in $F$. Let $E$ be another finite extension of $\mathbf{Q}_{p}$. A filtered $(\varphi, N, F, E)$-module (with descent data, i.e., $\operatorname{Gal}\left(F / \mathbf{Q}_{p}\right)$-action) is a free of finite rank $\left(F_{0} \otimes_{\mathbf{Q}_{p}} E\right)$-module $D$ endowed with

- the Frobenius endomorphism: an $F_{0}$-semi-linear, $E$-linear, bijective map $\varphi: D \rightarrow D$,

- the monodromy operator: an $\left(F_{0} \otimes_{\mathbf{Q}_{p}} E\right)$-linear, nilpotent endomorphism $N: D \rightarrow D$ which satisfies $N \varphi=p \varphi N$,

- an $F_{0}$-semi-linear, $E$-linear action of $\operatorname{Gal}\left(F / \mathbf{Q}_{p}\right)$ (the action on $F_{0}$ is via the projection to $\left.\operatorname{Gal}\left(F_{0} / \mathbf{Q}_{p}\right)\right)$, which commutes with the action of $\varphi$ and $N$,

- a decreasing filtration $\left(\mathrm{Fil}^{i} D_{F}\right)_{i \in \mathbf{Z}}$ of $\left(F \otimes_{\mathbf{Q}_{p}} E\right)$-submodules of $D_{F}=F \otimes_{F_{0}} D$ satisfying

$$
\mathrm{Fil}^{i} D_{F}=0 \text { for } i \gg 0 \text { and } \mathrm{Fil}^{i} D_{F}=D_{F} \text { for } i \ll 0
$$

and which are stable under the action of $\operatorname{Gal}\left(F / \mathbf{Q}_{p}\right)$.

Let $D$ be a filtered $(\varphi, N, F, E)$-module. Then by forgetting the $E$-module structure, $D$ is also a filtered $\left(\varphi, N, F, \mathbf{Q}_{p}\right)$-module. Let $d=\operatorname{dim}_{F_{0}} D$. Then $\Lambda_{F_{0}}^{d} D$ is a filtered $\left(\varphi, N, F, \mathbf{Q}_{p}\right)$-module of dimension 1 over $F_{0}$. Set

$$
t_{H}(D)=\max \left\{i \in \mathbf{Z} \mid \operatorname{Fil}^{i}\left(F \otimes_{F_{0}} \Lambda_{F_{0}}^{d} D\right) \neq 0\right\}, \quad t_{N}(D)=\operatorname{val}_{p}(\lambda),
$$

where for a non-zero element $x$ of $\Lambda_{F_{0}}^{d} D, \varphi(x)=\lambda x$, with $\lambda \in F_{0}^{\times}$. One says that $D$ is admissible (originally weakly admissible) if

- $t_{H}(D)=t_{N}(D)$, 
- for any $F_{0}$-submodule $D^{\prime}$ of $D$ stable by $\varphi$ and $N, t_{H}\left(D^{\prime}\right) \leq t_{N}\left(D^{\prime}\right)$, where $D_{F}^{\prime} \subset D_{F}$ is equipped with the induced filtration.

It turns out that the filtered $(\varphi, N, F, E)$-module $D$ is admissible if the second condition above is replaced by the following weaker condition (cf. [BM02, prop. 3.1.1.5]):

- for any $\left(F_{0} \otimes \mathbf{Q}_{p} E\right)$-submodule $D^{\prime}$ of $D$ stable by $\varphi$ and $N, t_{H}\left(D^{\prime}\right) \leq t_{N}\left(D^{\prime}\right)$, where again $D_{F}^{\prime} \subset D_{F}$ is equipped with the induced filtration.

2.2. Fontaine's rings. We wish to recall briefly the definition of some of Fontaine's rings of periods (cf. [Fon94b], [Ber04]). For $F$ a finite extension of $\mathbf{Q}_{p}$, we set $G_{F}=\operatorname{Gal}\left(\overline{\mathbf{Q}}_{p} / F\right)$, and set $G_{p}=G_{\mathbf{Q}_{p}}$. Fontaine's rings are topological $\mathbf{Q}_{p}$-algebras $\mathbf{B}$ equipped with an action of $G_{p}$ such that $\mathbf{B}^{G_{F}}$ is a field for every finite extension $F$ of $\mathbf{Q}_{p}$. For us the main example will be $\mathbf{B}_{\text {st }}$ which sits (non-canonically) between $\mathbf{B}_{\text {cris }}$ and $\mathbf{B}_{\mathrm{dR}}$.

Let

$$
\widetilde{\mathbf{E}}^{+}=\lim _{x \mapsto x} \mathcal{O}_{\mathbf{C}_{p}}=\left\{x=\left(x^{(0)}, x^{(1)}, \ldots,\right) \mid x^{(i)} \in \mathcal{O}_{\mathbf{C}_{p}},\left(x^{(i+1)}\right)^{p}=x^{(i)}\right\}
$$

Addition given by $(x+y)^{(i)}=\lim _{n \rightarrow \infty}\left(x^{(i+n)}+y^{(i+n)}\right)^{p^{n}}$ and componentwise multiplication turn $\widetilde{\mathbf{E}}^{+}$into a perfect ring of characteristic $p$. Let $\widetilde{\mathbf{A}}^{+}=W\left(\widetilde{\mathbf{E}}^{+}\right)$be the ring of Witt vectors, and let $\widetilde{\mathbf{B}}^{+}=\widetilde{\mathbf{A}}^{+}[1 / p]$. Elements of $\widetilde{\mathbf{A}}^{+}$, respectively $\widetilde{\mathbf{B}}^{+}$, may be written as $\sum p^{k}\left[x_{k}\right]$, where the sum is over $k \geq 0$, respectively $k \geq-n$ for some $n \geq 0$, and [ ] denotes the Teichmüller representative.

There is a ring homomorphism $\theta: \widetilde{\mathbf{B}}^{+} \rightarrow \mathbf{C}_{p}$ which maps $\sum p^{k}\left[x_{k}\right]$ to $\sum p^{k} x_{k}^{(0)}$, and $\operatorname{ker}(\theta)=(\omega)$ is a principal ideal. We let $\mathbf{B}_{\mathrm{dR}}^{+}$be the completion of $\widetilde{\mathbf{B}}^{+}$with respect to $\operatorname{ker}(\theta)$. So elements of $\mathbf{B}_{\mathrm{dR}}^{+}$ can be written as $\sum b_{n} \omega^{n}$ with $b_{n} \in \widetilde{\mathbf{B}}^{+}$.

Fix an element $\epsilon=\left(\epsilon^{(0)}, \epsilon^{(1)}, \ldots\right) \in \widetilde{\mathbf{E}}^{+}$where $\epsilon^{(n)}$ is a primitive $p^{n}$-th root of unity. Then $\theta(1-[\epsilon])=0$ and $t:=\log [\epsilon]$ converges as a series in $\mathbf{B}_{\mathrm{dR}}^{+}$. Define $\mathbf{B}_{\mathrm{dR}}=\mathbf{B}_{\mathrm{dR}}^{+}[1 / t]$.

Then $\mathbf{B}_{\mathrm{dR}}$ has a natural filtration given by $\mathrm{Fil}^{i} \mathbf{B}_{\mathrm{dR}}=t^{i} \mathbf{B}_{\mathrm{dR}}^{+}$. Also, under the natural Galois action, $\mathbf{B}_{\mathrm{dR}}^{G_{F}}=F$ for any finite extension $F$ of $\mathbf{Q}_{p}$.

Now set

$$
\mathbf{B}_{\text {cris }}^{+}=\left\{x=\sum b_{n} \frac{\omega^{n}}{n !} \in \mathbf{B}_{\mathrm{dR}}^{+} \mid b_{n} \in \widetilde{\mathbf{B}}^{+}, b_{n} \rightarrow 0\right\}
$$

where the convergence of the $b_{n}$ is in the $p$-adic topology. Finally set $\mathbf{B}_{\text {cris }}=\mathbf{B}_{\text {cris }}^{+}[1 / t]$. Then $\mathbf{B}_{\text {cris }}$ has a Frobenius map $\varphi: \mathbf{B}_{\text {cris }} \rightarrow \mathbf{B}_{\text {cris }}$, and $\mathbf{B}_{\text {cris }}^{G_{F}}=F_{0}$.

Now let $\mathbf{B}_{\text {st }}=\mathbf{B}_{\text {cris }}[Y]$ be the polynomial ring in one variable over $\mathbf{B}_{\text {cris }}$. Extend the Frobenius map to $\mathbf{B}_{\text {st }}$ by defining $\varphi(Y)=p Y$. There is also the monodromy operator $N=-\frac{d}{d Y}$ on $\mathbf{B}_{\text {st }}$ which satisfies $N \varphi=p \varphi N$. Finally the Galois action on $Y$ may be specified, and again one has $\mathbf{B}_{\mathrm{st}}^{G_{F}}=F_{0}$ ([Fon94b] $)$. Set $\tilde{p}=\left(p^{(0)}, p^{(1)}, \ldots\right) \in \widetilde{\mathbf{E}}^{+}$, where $p^{(n)}$ is a primitive $p^{n}$-th root of $p$. We will think of $\mathbf{B}_{\text {st }}$ as a subring of $\mathbf{B}_{\mathrm{dR}}$ by mapping $Y$ to $\log [\tilde{p}]$, though the definition of this last element depends on a choice, that of $\log _{p}(p)$, which we will take to be 0 . 
2.3. Potentially semi-stable representations. Let $E$ be a finite extension of $\mathbf{Q}_{p}$. We recall here that a representation $\rho: G_{p} \rightarrow \mathrm{GL}_{n}(E)=\mathrm{GL}(V)$ is said to be semi-stable over $F$ or $F$-semi-stable if the dimension of $D_{\mathrm{st}, F}(V)=\left(\mathbf{B}_{\mathrm{st}} \otimes \mathbf{Q}_{p} V\right)^{G_{F}}$ over $F_{0}=\mathbf{B}_{\mathrm{st}}^{G_{F}}$ is equal to the dimension of $V$ over $\mathbf{Q}_{p}$ (in general it may be less). If such an $F$ exists, $\rho$ is said to be a potentially semi-stable representation.

Potentially semi-stable representations are known to be Hodge-Tate. We recall the definition of the associated Hodge-Tate weights. Let

$$
\left(\mathbf{C}_{p} \otimes_{\mathbf{Q}_{p}} V\right)\{i\}=\left\{x \in \mathbf{C}_{p} \otimes_{\mathbf{Q}_{p}} V \mid g(x)=\nu^{i}(g) x, \forall g \in G_{p}\right\},
$$

where $\nu$ is the $p$-adic cyclotomic character and $G_{p}$ acts on $\mathbf{C}_{p}$ and on $V$. This is an $E$-vector space of finite dimension. By [Fon94a, §3], there is a $G_{p}$-equivariant isomorphism

$$
\bigoplus_{i \in \mathbf{Z}} \mathbf{C}_{p} \otimes \mathbf{Q}_{p}\left(\mathbf{C}_{p} \otimes \mathbf{Q}_{p} V\right)\{i\} \stackrel{\sim}{\longrightarrow} \mathbf{C}_{p} \otimes \mathbf{Q}_{p} V
$$

of $\left(\mathbf{C}_{p} \otimes \mathbf{Q}_{p} E\right)$-modules (where the $G_{p}$-action is $\mathbf{C}_{p}$-semi-linear and $E$-linear). The integers $i$ such that $\left(\mathbf{C}_{p} \otimes_{\mathbf{Q}_{p}} V\right)\{i\} \neq 0$, counted with multiplicity $\operatorname{dim}_{E}\left(\mathbf{C}_{p} \otimes_{\mathbf{Q}_{p}} V\right)\{i\}$, are by definition the Hodge-Tate weights of the representation $V$.

It is known by the fundamental work of Colmez and Fontaine [CF00] that there is an equivalence of categories between the $F$-semi-stable representations $\rho: G_{p} \rightarrow \mathrm{GL}_{n}(E)$ with Hodge-Tate weights in $\{0, \ldots, k-1\}$ and the admissible filtered $(\varphi, N, F, E)$-module $D$ of rank $n$ over $F_{0} \otimes_{\mathbf{Q}_{p}} E$ such that $\mathrm{Fil}^{1-k}\left(D_{F}\right)=D_{F}$ and $\mathrm{Fil}^{1}\left(D_{F}\right)=0$. The equivalence of categories is induced by the functor $D_{\mathrm{st}, F}$ defined above. The Frobenius, monodromy, and filtration on $\mathbf{B}_{\mathrm{st}}$ induce the corresponding objects on $D_{\mathrm{st}, F}(V)$. There is also an induced action of $\operatorname{Gal}\left(F / \mathbf{Q}_{p}\right)$ on $D_{\mathrm{st}, F}(V)$.

We remark that the jumps in the filtration on $D_{F}=F \otimes_{F_{0}} D_{\text {st }, F}(V)$ are the negatives of the Hodge-Tate weights of $V$. That is, if $h$ is a Hodge-Tate weight, one has $\mathrm{Fil}^{-h}\left(D_{F}\right) \neq \mathrm{Fil}^{-h+1}\left(D_{F}\right)$.

2.4. Weil-Deligne representations. Let $\rho: G_{p} \rightarrow \mathrm{GL}_{n}(E)$ be an $F$-semi-stable representation. Let us recall the definition of the Weil-Deligne representation associated to $\rho$. Let $W_{p}$ be the Weil group of $\mathbf{Q}_{p}$ and $W_{F}$ the Weil group of $F$. Recall that $W_{p} / W_{F}=\operatorname{Gal}\left(F / \mathbf{Q}_{p}\right)$. One equips $D_{\text {st }, F}(V)$ with an action of $W_{p}$ by making

$$
g \in W_{p} \text { act by }\left(g \bmod W_{F}\right) \circ \varphi^{-\alpha(g)},
$$

where the image of $g$ in $\operatorname{Gal}\left(\overline{\mathbf{F}}_{p} / \mathbf{F}_{p}\right)$ is the $\alpha(g)$-th power of the arithmetic Frobenius at $p$. Since both $\operatorname{Gal}\left(F / \mathbf{Q}_{p}\right)$ and $\varphi$ act $F_{0}$-semi-linearly, evidently $W_{F}$ acts $F_{0}$-linearly.

Now assume that $E$ contains $F$. (To define the Weil-Deligne representation one need only assume that $E$ contains $F_{0}$, but the stronger assumption $F \subset E$ will be used later.) Then

$$
D_{\mathrm{st}, F}(V) \simeq \prod_{\sigma: F_{0} \hookrightarrow E} D_{\sigma, F},
$$

where $D_{\sigma, F}=D_{\mathrm{st}, F}(V) \otimes_{\left(F_{0} \otimes \mathbf{Q}_{p} E, \sigma\right)} E$ is an $E$-vector space of dimension $n$ with an induced action of $\left(W_{p}, N\right)$. In particular the $D_{\sigma, F}$ are Weil-Deligne representations. By [BM02, lemme 2.2.1.2] 
the isomorphism class of $D_{\sigma, F}$ is independent of the choice of $\sigma$ (and $F$ ) and is by definition the Weil-Deligne representation $W D(\rho)$ attached to $\rho$. Note that if inertia acts on a vector in $D_{\sigma, F}$ by $\chi$, then under the identification (2.2) inertia acts on the corresponding vector in $D_{\mathrm{st}, F}(V)$ by $1 \otimes \chi$.

2.5. Galois types. Let $I_{p}$ be the inertia group at $p$. A Galois type $\tau$ of degree $n$ is an equivalence class of representations

$$
\tau: I_{p} \rightarrow \mathrm{GL}_{n}\left(\overline{\mathbf{Q}}_{p}\right)
$$

with open kernel which extend to representations of the Weil group $W_{p}$ ([BCDT, $\left.\left.\S 1\right]\right)$. An $F$-semistable representation $\rho: G_{p} \rightarrow \mathrm{GL}_{n}\left(\overline{\mathbf{Q}}_{p}\right)$ has Galois type $\tau$ if $\left.W D(\rho)\right|_{I_{p}} \simeq \tau$. Write $\mathbf{Q}_{p^{m}}$ for the unique unramified extension of $\mathbf{Q}_{p}$ of degree $m$ and $\mathbf{Z}_{p^{m}}$ for its ring of integers. Also write $W_{p^{m}}$ for the Weil group of $\mathbf{Q}_{p^{m}}$. Let us recall the following classical lemma:

Lemma 2.1. Assume $p>2$. Let $\tau$ be a Galois type of degree 2. Then $\tau$ has one of the following forms:

(1) $\left.\left.\tau \simeq \chi_{1}\right|_{I_{p}} \oplus \chi_{2}\right|_{I_{p}}$, where $\chi_{1}, \chi_{2}$ are characters of $W_{p}$ finite on $I_{p}$,

(2) $\left.\tau \simeq \operatorname{Ind}_{W_{p^{2}}}^{W_{p}}(\chi)\right|_{I_{p}}=\left.\left.\chi\right|_{I_{p}} \oplus \chi^{\sigma}\right|_{I_{p}}$, where $\chi$ is a character of $W_{p^{2}}$ finite on $I_{p}$ which does not extend to $W_{p}$, and $\sigma$ is the generator of $\operatorname{Gal}\left(\mathbf{Q}_{p^{2}} / \mathbf{Q}_{p}\right)$,

(3) $\left.\tau \simeq \operatorname{Ind}_{W_{K}}^{W_{p}}(\chi)\right|_{I_{p}}$, where $K$ is a ramified quadratic extension of $\mathbf{Q}_{p}$, $\chi$ is a character of $W_{K}$, finite on $I_{K}$, such that $\left.\chi\right|_{I_{K}}$ does not extend to $I_{p}$.

In the first two cases $\tau$ is reducible, and in the third case $\tau$ is irreducible.

Again let $p>2$. Let $\rho: G_{p} \rightarrow \mathrm{GL}_{2}(E)$ be a potentially semi-stable representation and let $\tau=\left.W D(\rho)\right|_{I_{p}}$ be its Galois type. Here are some useful facts ([BM02, §2]).

Lemma 2.2. The potentially semi-stable representation $\rho$ is semi-stable over $F$ if and only if $\left.\tau\right|_{I_{F}}$ is trivial.

Lemma 2.3. If $\rho$ is not potentially crystalline (that is, $N \neq 0$ ), then $\tau$ is a scalar.

Proof. Suppose that $\rho$ becomes semi-stable over $F$, and let $D=D_{\text {st }, F}(V)$ be the associated filtered $(\varphi, N, F, E)$-module. The action of $g \in W_{p}$ satisfies $g N=p^{\alpha(g)} N g$. Hence $W D(\rho)$ stabilizes ker $N$ and so is reducible. In particular the action of $I_{p}$ is reducible and therefore a direct sum of two characters. Since $N$ commutes with $I_{p}$, these two characters must be the same on $I_{p}$.

There are therefore three possibilities:

- $N \neq 0$ and $\tau$ is a scalar (special or Steinberg case),

- $N=0$ and $\tau$ is as in (1) of Lemma 2.1 (principal series case),

- $N=0$ and $\tau$ is as in (2) or (3) of Lemma 2.1 (supercuspidal case). 
2.6. Question. Given a potentially semi-stable representation $\rho: G_{p} \rightarrow \mathrm{GL}_{2}(E)$, one may associate to it

- its Galois type, i.e., the Weil-Deligne representation $\left.W D(\rho)\right|_{I_{p}}$, and,

- its Hodge-Tate weights.

The following question is of interest: given a Galois type $\tau$ of degree 2 and an integer $k>1$, what are the (isomorphism classes of) potentially semi-stable representations $\rho: G_{p} \rightarrow \mathrm{GL}_{2}(E)$ having type $\tau$ and Hodge-Tate weights 0 and $k-1$ ? More specifically, which of these representations occur as the local components of Galois representations attached to elliptic modular cusp forms (cf. [Bre01, p. 32])?

To answer these questions it suffices, by what has been said above, to write down the corresponding filtered modules. In this paper we will essentially classify rank two filtered modules with coefficients when $p>2$ and $F \subset E$, concentrating on the new features that arise when $E \neq \mathbf{Q}_{p}$ (and $F \neq \mathbf{Q}_{p}$ ). For the filtered modules arising from cusp forms, we will indicate (in some cases) how the filtered modules depend on the underlying form.

Our arguments below are broken down according to the type $\tau$ of the representation. For the remainder of this article we assume that $F \subset E, p>2$, and $k>1$.

\section{RANK TWO FILTERED MODULES}

3.1. Special case. In this case $\tau$ is a scalar. No new issues arise when one has coefficients. We assume $\tau=1$ and briefly recall the construction of the filtered module in this case (see also [Maz94, $\S 9$ ], [FM95, p. 65]). We have $F=F_{0}=\mathbf{Q}_{p}$. Since $N \neq 0$ there exists a basis $e_{1}, e_{2}$ of $D=D_{\mathrm{st}, \mathbf{Q}_{p}}(V)$ such that $N\left(e_{1}\right)=e_{2}$ and $N\left(e_{2}\right)=0$. Since $N \varphi=p \varphi N$ we must have that $\varphi\left(e_{1}\right)=\frac{p}{\alpha} e_{1}+c e_{2}$ and $\varphi\left(e_{2}\right)=\frac{1}{\alpha} e_{2}$, with $c, \alpha \in E$. Replacing $e_{1}$ with a linear combination of $e_{1}$ and a scalar multiple of $e_{2}$, we may assume $c=0$. Since $D$ is admissible, $t_{H}(D)=t_{N}(D)$, i.e., $\operatorname{val}_{p}\left(\frac{\varphi\left(e_{1} \wedge e_{2}\right)}{e_{1} \wedge e_{2}}\right)=\operatorname{val}_{p}\left(\frac{p}{\alpha^{2}}\right)=1-k$. So $\operatorname{val}_{p}(\alpha)=k / 2$.

Assume Fil $^{0} D_{F}=$ Fil $^{0} D=E\left(a e_{1}+b e_{2}\right)$. The only sub- $(\varphi, N)$-module of $D$ is $D_{2}=\left\langle e_{2}\right\rangle$. If $a=0$, then the admissibility condition shows that $t_{H}\left(D_{2}\right) \leq t_{N}\left(D_{2}\right)$, so that $0 \leq-\operatorname{val}_{p}(\alpha)$, which is clearly impossible. If $a \neq 0, D$ is admissible.

The admissible filtered $(\varphi, N, F, E)$-module associated to $\rho$ is (up to isomorphism) given by $D_{\mathrm{st}}=$ $E e_{1} \oplus E e_{2}$ with

$$
D_{\mathrm{st}}=\left\{\begin{array}{ccc}
\varphi\left(e_{1}\right) & = & \frac{p}{\alpha} e_{1} \\
\varphi\left(e_{2}\right) & = & \frac{1}{\alpha} e_{2} \\
\operatorname{val}_{p}(\alpha) & = & k / 2 \\
N\left(e_{1}\right) & = & e_{2} \\
N\left(e_{2}\right) & = & 0 \\
\operatorname{Fil}^{0} D & = & E\left(e_{1}-\mathfrak{L} e_{2}\right) \\
\mathfrak{L} & \in & E .
\end{array}\right.
$$


If $f=\sum_{n=1}^{\infty} a_{n} q^{n}$ is a cuspidal newform of weight $k \geq 2$, level $N$ and character $\epsilon$, and $p \| N$ but is coprime to the conductor of $\epsilon$, then by work of Faltings the associated local Galois representation $\left.\rho_{f}\right|_{G_{p}}: G_{p} \rightarrow \mathrm{GL}_{2}(E)$ is known to be semi-stable [Maz94, §12]. The associated filtered module $D_{\text {st }}\left(\left.\rho_{f}\right|_{G_{p}}\right)$ is as above with $\alpha=p a_{p}$ (see [Bre01, pp. 31-32], where the normalizations are slightly different from ours). It is known that $a_{p}^{2}=\epsilon(p) p^{k-2}\left[\right.$ Miy89, Thm. 4.6.17(ii)] so $\operatorname{val}_{p}(\alpha / p)=k / 2-1$ and $\operatorname{val}_{p}(\alpha)=k / 2$. The quantity $\mathfrak{L}$ is then by definition the $\mathfrak{L}$-invariant of $f$ (using the definition of the $\mathfrak{L}$-invariant due to Mazur in [Maz94]).

3.2. Ramified principal series case. We now assume $\left.\left.\tau \simeq \chi_{1}\right|_{I_{p}} \oplus \chi_{2}\right|_{I_{p}}$ (and $\left.N=0\right)$. In this case new features may arise since neither $F$ nor $E$ is necessarily $\mathbf{Q}_{p}$.

Let us say we are in the unramified principal series case if both characters $\chi_{1}$ and $\chi_{2}$ are trivial on $I_{p}$, that is, $\tau=1$. In this case $F=\mathbf{Q}_{p}$ and the corresponding filtered module already occurs in the literature (cf. [Bre01, pp. 30-32], where the normalizations are slightly different from ours). If $\tau \neq 1$ we shall say we are in the ramified principal series case. We shall assume $\left.\left(\chi_{1} \chi_{2}^{-1}\right)\right|_{I_{p}} \neq \mathrm{Id}$ and compute the filtered modules in this case.

We note $\rho$ is potentially crystalline. The characters $\left.\chi_{i}\right|_{I_{p}}$ factor through $\operatorname{Gal}\left(\mathbf{Q}_{p}^{\mathrm{nr}}\left(\zeta_{p^{m}}\right) / \mathbf{Q}_{p}^{\mathrm{nr}}\right) \simeq$ $\operatorname{Gal}\left(\mathbf{Q}_{p}\left(\zeta_{p^{m}}\right) / \mathbf{Q}_{p}\right)$ for $m \geq 1$. Hence we may take $F=\mathbf{Q}_{p}\left(\zeta_{p^{m}}\right)$ and $F_{0}=\mathbf{Q}_{p}$.

Now write $D=E e_{1} \oplus E e_{2}$, where $g \in \operatorname{Gal}\left(F / \mathbf{Q}_{p}\right)$ acts by $\chi_{n}$ on $e_{n}$ for $n=1,2$. Write

$$
\varphi\left(e_{1}\right)=q e_{1}+r e_{2}, \quad \varphi\left(e_{2}\right)=s e_{1}+t e_{2},
$$

where the coefficients $q, r, s, t \in E$. Since $\varphi$ commutes with the action of $\operatorname{Gal}\left(F / \mathbf{Q}_{p}\right), r=0=s$, so that $\varphi\left(e_{1}\right)=\frac{1}{\alpha} e_{1}$ and $\varphi\left(e_{2}\right)=\frac{1}{\beta} e_{2}$, for some $\alpha, \beta \in E$. Since $D$ is admissible, $\operatorname{val}_{p}\left(\frac{\varphi\left(e_{1} \wedge e_{2}\right)}{e_{1} \wedge e_{2}}\right)=1-k$, whence $\operatorname{val}_{p}(\alpha)+\operatorname{val}_{p}(\beta)=k-1$. Now $D_{n}=\left\langle e_{n}\right\rangle$, for $n=1,2$, are sub $\varphi$-modules, and since $t_{H}\left(D_{n}\right)$ is 0 or $1-k$, which in either case is $\geq 1-k$, the admissibility condition $t_{H}\left(D_{n}\right) \leq t_{N}\left(D_{n}\right)$ shows that $\operatorname{val}_{p}(\alpha), \operatorname{val}_{p}(\beta) \leq k-1$. So we have (without loss of generality) that

$$
0 \leq \operatorname{val}_{p}(\alpha) \leq \operatorname{val}_{p}(\beta) \leq k-1
$$

There are two cases to consider, depending on the valuations of $\alpha$ and $\beta$, or put another way, depending on the slopes of the Newton polygon of $\varphi$.

I) Ordinary Case: $\operatorname{val}_{p}(\alpha)=0, \operatorname{val}_{p}(\beta)=k-1$.

We make a table that summarizes the various possibilities for admissibility of the two sub $\varphi-$ modules $D_{n}=\left\langle e_{n}\right\rangle$ depending on various choices for the filtration. Savitt has observed that any $\left(F \otimes_{F_{0}} E\right)$-submodule of a filtered module with descent data (i.e., $\operatorname{Gal}\left(F / \mathbf{Q}_{p}\right)$-action) has to be free [Sav05, Lemma 2.1]. Thus $\operatorname{Fil}^{0}\left(D_{F}\right)$ is spanned by a vector of the form $x e_{1}+y e_{2}$, where $x$,

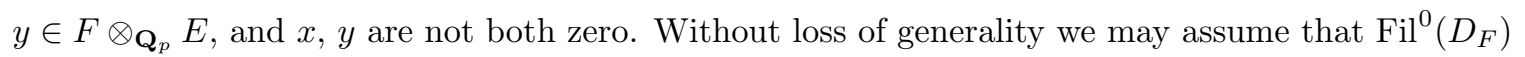
is spanned by either of the axes $e_{1}$ or $e_{2}$, or some linear combination $v=x e_{1}+y e_{2}$, where $x, y$ are non-zero elements of $F \otimes \mathbf{Q}_{p} E$. 


\begin{tabular}{|c||c|c|c||c|c|c||c||}
\hline $\operatorname{Fil}^{0}\left(D_{F}\right)$ & $t_{H}\left(D_{1}\right)$ & $t_{N}\left(D_{1}\right)$ & $D_{1}$ ADM? & $t_{H}\left(D_{2}\right)$ & $t_{N}\left(D_{2}\right)$ & $D_{2}$ ADM? & Type \\
\hline$e_{1}$ & 0 & 0 & Yes & $1-k$ & $1-k$ & Yes & $D=D_{1} \oplus D_{2}$ \\
\hline$e_{2}$ & $1-k$ & 0 & No & 0 & $1-k$ & No & $D$ not admissible \\
\hline$v$ & $1-k$ & 0 & No & $1-k$ & $1-k$ & Yes & $D_{2} \subset D$ \\
\hline
\end{tabular}

We shall refer to the first and third cases in the table above as the ordinary split case $\left(D_{\text {ord-split }}\right)$, and the ordinary non-split case ( $D_{\text {ord-non-split }}$ ) respectively. In both these cases the corresponding Galois representation is reducible. In the first case it is decomposable or split, whereas in the third case it is indecomposable or non-split, whence the terminology. In the second case in the table above $D$ is not admissible since $t_{H}\left(D_{2}\right)>t_{N}\left(D_{2}\right)$ if $k>1$.

II) Non-Ordinary Case: $0<\operatorname{val}_{p}(\alpha) \leq \operatorname{val}_{p}(\beta)<k-1$.

In this case, if $\mathrm{Fil}^{0}\left(D_{F}\right)$ were spanned by $e_{1}$ or $e_{2}$, then the admissibility condition for $D$ would imply that either $0 \leq-\operatorname{val}_{p}(\alpha)$ or $0 \leq-\operatorname{val}_{p}(\beta)$, both of which are clearly impossible. $\operatorname{So~}_{\operatorname{Fil}^{0}}\left(D_{F}\right)$ must be $\left(F \otimes_{\mathbf{Q}_{p}} E\right) v$. But now $D$ does not have admissible subobjects (one clearly sees that neither $D_{1}$ nor $D_{2}$ is admissible).

As already noted above $\operatorname{Fil}^{0}\left(D_{F}\right)$ is a free $\left(F \otimes_{\mathbf{Q}_{p}} E\right)$-module of rank 1. Let us make some remarks about the vector $v$ which spans $\operatorname{Fil}^{0}\left(D_{F}\right)$ when this module is not one of the two axes. A first requirement is that the line $\left(F \otimes \mathbf{Q}_{p} E\right) \cdot v$ should be stable under $\operatorname{Gal}\left(F / \mathbf{Q}_{p}\right)$. It is not immediately evident that such a stable line exists. As it turns out such a line does exist and moreover is unique (up to isomorphism). In order to show this we start with the following useful lemma.

Lemma 3.1. Every Galois stable line $\left(F \otimes_{\mathbf{Q}_{p}} E\right) \cdot v$ in $D_{F}$ is spanned by an invariant vector $v$.

Proof. Let $G=\operatorname{Gal}\left(F / \mathbf{Q}_{p}\right)$. Then $G=\left(\mathbf{Z} / p^{m}\right)^{\times}$is a cyclic group. We first claim that $\mathrm{H}^{1}\left(G,\left(F \otimes \mathbf{Q}_{p}\right.\right.$ $\left.E)^{\times}\right)=0$. Let $g$ be a generator of $G$. Let $N=1+g+g^{2}+\cdots+g^{r-1}$, where $r=\phi\left(p^{m}\right)=p^{m-1}(p-1)$ is the order of $G$ and let $D=g-1$. Then for any $G$-module $A$ one knows

$$
\mathrm{H}^{1}(G, A)=\frac{\operatorname{ker}(N: A \rightarrow A)}{\operatorname{Im}(D: A \rightarrow A)} .
$$

Apply this to $A=\left(F \otimes_{\mathbf{Q}_{p}} E\right)^{\times}=\prod_{\sigma} E^{\times}$noting that $g$ acts by cyclically permuting the factors. Then $\left(x_{0}, x_{1}, x_{2}, \ldots, x_{r-1}\right)$ lies in the kernel of $N$ iff $\prod x_{i}=1$. Similarly $\left(x_{0}, x_{1}, x_{2}, \ldots, x_{r-1}\right)$ lies in the image of $D$ iff it has the form $\left(\frac{y_{1}}{y_{0}}, \frac{y_{2}}{y_{1}}, \frac{y_{3}}{y_{2}}, \ldots, \frac{y_{0}}{y_{r-1}}\right)$. Taking $\left(y_{0}, y_{1}, y_{2}, \ldots, y_{r-1}\right)=$ $\left(1, x_{0}, x_{0} x_{1}, x_{0} x_{1} x_{2}, \ldots, x_{0} x_{1} \cdots x_{r-2}\right)$ we are done.

We now show that every Galois stable line in $D_{F}$ is spanned by an invariant vector. Say $\left(F \otimes \mathbf{Q}_{p}\right.$ $E) \cdot v$ is stable by $G=\operatorname{Gal}\left(F / \mathbf{Q}_{p}\right)$. Then for $h \in G$ we have $h \cdot v=c_{h} v$ for some $c_{h} \in\left(F \otimes_{\mathbf{Q}_{p}} E\right)^{\times}$. One easily checks that $c_{h} \in \mathrm{Z}^{1}\left(G,\left(F \otimes_{\mathbf{Q}_{p}} E\right)^{\times}\right)$is a 1-cocycle. Since $\mathrm{H}^{1}\left(G,\left(F \otimes_{\mathbf{Q}_{p}} E\right)^{\times}\right)=0$ we see that $c_{h}$ is a 1-coboundary. Say $c_{h}=c / h(c)$ for some $c \in\left(F \otimes_{\mathbf{Q}_{p}} E\right)^{\times}$. Replacing $v$ by $c v$ we see that we may assume that $c_{h}=1$ and that $v$ is invariant under $G$. 
Remark 3.2. As pointed out to us by D. Prasad, the vanishing of the first cohomology group in the proof above is in fact a special case of a general vanishing result: namely $\mathrm{H}^{i}\left(G,\left(F \otimes \mathbf{Q}_{p} E\right)^{\times}\right)=0$ for any finite group $G=\operatorname{Gal}\left(F / \mathbf{Q}_{p}\right)$ and any $i>0$. Indeed $\left(F \otimes \mathbf{Q}_{p} E\right)^{\times}=\prod E^{\times}$with the permutation action of $G$ is just $\operatorname{Ind}_{H}^{G}\left(E^{\times}\right)$, the $G$-module induced from the trivial subgroup $H=\{1\}$ to $G$ of the trivial $H$-representation $E^{\times}$. By Shapiro's lemma, $\mathrm{H}^{i}\left(G, \operatorname{Ind}_{H}^{G}\left(E^{\times}\right)\right)=\mathrm{H}^{i}\left(H, E^{\times}\right)=0$ if $i>0$ since $H$ is trivial.

By the lemma we are reduced to constructing Galois invariant vectors $v$ in $D_{F}$. Using Gauss sum-like elements we now prove the following proposition.

Proposition 3.3. Let $F=\mathbf{Q}_{p}\left(\zeta_{p^{m}}\right)$. There exist explicit elements $x, y \in F \otimes \mathbf{Q}_{p} E$, both non-zero, such that

$$
v=x e_{1}+y e_{2}
$$

is invariant by $\operatorname{Gal}\left(F / \mathbf{Q}_{p}\right)$. Every Galois stable line in $D_{F}$ (which is not one of two axes) is of the form $\left(F \otimes_{\mathbf{Q}_{p}} E\right) \cdot\left(c x e_{1}+d y e_{2}\right)$, for some non-zero $c$, $d$ in $E$. In particular up to an isomorphism obtained by scaling $e_{1}$ and $e_{2}$ we may assume that $\mathrm{Fil}^{0}\left(D_{F}\right)=\left(F \otimes_{\mathbf{Q}_{p}} E\right) \cdot\left(x e_{1}+y e_{2}\right)$.

Proof. Write $\operatorname{Gal}\left(F / \mathbf{Q}_{p}\right)=\Delta \times \Gamma$, where $\Delta=(\mathbf{Z} / p \mathbf{Z})^{\times}$and $\Gamma=\mathbf{Z} / p^{m-1} \mathbf{Z}$. These groups are also cyclic: write $\delta$ for a generator of $\Delta$, and $\gamma$ for a generator of $\Gamma$ corresponding to $1+p$ under the identification $\operatorname{Gal}\left(F / \mathbf{Q}_{p}\right)=\left(\mathbf{Z} / p^{m}\right)^{\times}$. Note that every character $\chi$ of $\operatorname{Gal}\left(F / \mathbf{Q}_{p}\right)$ is of the form

$$
\chi=\omega^{i} \chi_{\zeta^{a}}, \quad \text { with } i, a \in \mathbf{Z}, \quad 0 \leq i<p-1, \quad 0 \leq a \leq p^{m-1}-1,
$$

where $\omega$ is the Teichmüller character on $\Delta$, and $\chi_{\zeta^{a}}$ is the character on $\Gamma$ which maps $\gamma$ to $\zeta^{a}$, where $\zeta=\zeta_{p^{m-1}}$ is a primitive $p^{m-1}$-th root of unity. Thus we have $\chi_{\left.1\right|_{I_{p}}}=\omega^{i} \chi_{\zeta^{a}}$ and similarly $\chi_{\left.2\right|_{I_{p}}}=\omega^{j} \chi_{\zeta^{b}}$ for integers $0 \leq i, j<p-1$, and $0 \leq a, b<p^{m-1}-1$.

Suppose that the vector $x^{\prime} e_{1}+y^{\prime} e_{2}$ with $x^{\prime}, y^{\prime}$ in $F \otimes \mathbf{Q}_{p} E$ is $\operatorname{Gal}\left(F / \mathbf{Q}_{p}\right)$ invariant. Then it must be invariant by $\Gamma$. Since $\operatorname{Gal}\left(F / \mathbf{Q}_{p}\right)$ acts by $1 \otimes \chi_{n}$ on $e_{n}$ for $n=1,2$, we must have

$$
\gamma\left(x^{\prime}\right)=\left(1 \otimes \zeta_{p^{m-1}}^{-a}\right) x^{\prime} \quad \text { and } \quad \gamma\left(y^{\prime}\right)=\left(1 \otimes \zeta_{p^{m-1}}^{-b}\right) y^{\prime} .
$$

After some amount of searching we take

$$
x^{\prime}=\sum_{r=0}^{p^{m-1}-1} \zeta_{p^{m}}^{(1+p)^{r}} \otimes \zeta_{p^{m-1}}^{r a} \quad \text { and } \quad y^{\prime}=\sum_{r=0}^{p^{m-1}-1} \zeta_{p^{m}}^{(1+p)^{r}} \otimes \zeta_{p^{m-1}}^{r b} .
$$

Note that the conditions (3.1) are satisfied since $\gamma\left(\zeta_{p^{m}}^{(1+p)^{r}}\right)=\zeta_{p^{m}}^{(1+p)^{(r+1)}}$. Moreover, we see that $x^{\prime}$ and $y^{\prime}$ are unique up to multiplication by an element of $\mathbf{Q}_{p}\left(\zeta_{p}\right) \otimes 1$ or an element of $1 \otimes E$. This follows from a dimension count of the eigenspaces of the $E$-linear operator $\gamma$ on $\prod_{\sigma} E=F \otimes \mathbf{Q}_{p} E$. In any case, we have

$$
\gamma\left(x^{\prime} e_{1}\right)=x^{\prime} e_{1} \quad \text { and } \quad \gamma\left(y^{\prime} e_{2}\right)=y^{\prime} e_{2} .
$$


Now taking an average over $\Delta$, let

$$
x^{\prime \prime}=\sum_{s \in(\mathbf{Z} / p \mathbf{Z})^{\times}} \delta^{s}\left(x^{\prime}\right) \quad \text { and } \quad y^{\prime \prime}=\sum_{s \in(\mathbf{Z} / p \mathbf{Z})^{\times}} \delta^{s}\left(y^{\prime}\right) .
$$

A check shows that $x^{\prime \prime}$ and $y^{\prime \prime}$ are non-zero elements in $F \otimes_{\mathbf{Q}_{p}} E$. Moreover, by construction, $\Delta$ fixes both $x^{\prime \prime}$ and $y^{\prime \prime}$. Now let $\pi \in F$ be such that $\pi^{p-1}+p=0$. So $\mathbf{Q}_{p}(\pi)=\mathbf{Q}_{p}\left(\zeta_{p}\right)$ and $\delta(\pi)=\omega(\delta) \pi$. We set $x=\pi^{-i} x^{\prime \prime}$ and $y=\pi^{-j} y^{\prime \prime}$. Again by construction,

$$
\delta\left(x e_{1}\right)=x e_{1} \quad \text { and } \quad \delta\left(y e_{2}\right)=y e_{2} .
$$

Also $\gamma$ continues to fix the quantities $x e_{1}$ and $y e_{2}$.

It follows that $v=x e_{1}+y e_{2}$ is invariant under $\operatorname{Gal}\left(F / \mathbf{Q}_{p}\right)=\Delta \times \Gamma$. Also $x$ and $y$ are unique up to multiplication by elements of $1 \otimes E$. Now $\operatorname{Fil}^{0}\left(D_{F}\right)$ is a $\operatorname{Gal}\left(F / \mathbf{Q}_{p}\right)$ stable line in $D_{F}$ (which is not one of the two axes). It follows from Lemma 3.1 that $\operatorname{Fil}^{0}\left(D_{F}\right)=\left(F \otimes_{\mathbf{Q}_{p}} E\right)\left(c x e_{1}+\right.$ dye $\left._{2}\right)$ for some non-zero elements $c, d$ in $1 \otimes E$. After scaling $e_{1}$ and $e_{2}$ (this induces an isomorphism of the filtered module $D$ ) we see that we may assume $\operatorname{Fil}^{0}\left(D_{F}\right)=\left(F \otimes_{\mathbf{Q}_{p}} E\right)\left(x e_{1}+y e_{2}\right)$. This proves the proposition.

Remark 3.4. The referee has pointed out that the construction of the elements $x$ and $y$ can be simplified as follows. By the normal basis theorem, choose $\theta \in F$ such that the conjugates of $\theta$ are a basis for $F$. Then every element of $F \otimes_{\mathbf{Q}_{p}} E$ can be written uniquely as $\sum g(\theta) \otimes a_{g}$, where the sum is over $g \in \operatorname{Gal}\left(F / \mathbf{Q}_{p}\right)$. If $\operatorname{Gal}\left(F / \mathbf{Q}_{p}\right)$ acts on such an element via $1 \otimes \chi_{1}^{-1}$, then $a_{h^{-1} g}=\chi_{1}^{-1}(h) a_{g}$ for all $h, g \in \operatorname{Gal}\left(F / \mathbf{Q}_{p}\right)$. Taking $g=1$ in this relation we obtain $a_{h}=\chi_{1}(h) a_{1}$, for all $h \in \operatorname{Gal}\left(F / \mathbf{Q}_{p}\right)$. Thus the element above must be a scalar multiple of $\sum g(\theta) \otimes \chi_{1}(g)$ by an element of $1 \otimes E$. Then define $x=\sum g(\theta) \otimes \chi_{1}(g)$, and define $y$ similarly using $\chi_{2}$ instead of $\chi_{1}$.

Let $x$ and $y$ be the elements constructed in the proposition. To summarize, the admissible filtered $(\varphi, N, F, E)$-modules associated to $\rho$ are given by $D=E e_{1} \oplus E e_{2}$ with

$$
D_{\text {ord-split }}=\left\{\begin{array}{cccc}
\varphi\left(e_{1}\right) & = & \frac{1}{\alpha} e_{1} \\
\varphi\left(e_{2}\right) & = & \frac{1}{\beta} e_{2} \\
\alpha, \beta & \in & E, & \operatorname{val}_{p}(\alpha)=0, \operatorname{val}_{p}(\beta)=k-1 \\
N & = & 0 \\
g\left(e_{1}\right) & = & \chi_{1}(g) e_{1} \\
g\left(e_{2}\right) & = & \chi_{2}(g) e_{2} \\
\operatorname{Fil}^{0}\left(D_{F}\right) & = & & \left(F \otimes \mathbf{Q}_{p} E\right) e_{1}
\end{array}\right.
$$




$$
\begin{aligned}
& D_{\text {ord-non-split }}=\left\{\begin{array}{cccc}
\varphi\left(e_{1}\right) & = & \frac{1}{\alpha} e_{1} \\
\varphi\left(e_{2}\right) & = & \frac{1}{\beta} e_{2} \\
\alpha, \beta & \in & E, & \operatorname{val}_{p}(\alpha)=0, \operatorname{val}_{p}(\beta)=k-1 \\
N & = & 0 \\
g\left(e_{1}\right) & = & \chi_{1}(g) e_{1} \\
g\left(e_{2}\right) & = & \chi_{2}(g) e_{2} \\
\operatorname{Fil}^{0}\left(D_{F}\right)= & & \left(F \otimes \mathbf{Q}_{p} E\right)\left(x e_{1}+y e_{2}\right)
\end{array}\right. \\
& D_{\text {non-ord }}=\left\{\begin{array}{cccc}
\varphi\left(e_{1}\right) & = & \frac{1}{\alpha} e_{1} \\
\varphi\left(e_{2}\right) & = & \frac{1}{\beta} e_{2} \\
\alpha, \beta & \in & E, & 0<\operatorname{val}_{p}(\alpha) \leq \operatorname{val}_{p}(\beta)<k-1 \\
N & = & 0 \\
g\left(e_{1}\right)= & \chi_{1}(g) e_{1} \\
g\left(e_{2}\right) & = & \chi_{2}(g) e_{2} \\
\operatorname{Fil}^{0}\left(D_{F}\right) & = & & \left(F \otimes \mathbf{Q}_{p} E\right)\left(x e_{1}+y e_{2}\right) .
\end{array}\right.
\end{aligned}
$$

These filtered modules arise frequently in nature. Indeed assume that

- $f=\sum_{n=1}^{\infty} a_{n} q^{n}$ is a cuspidal newform of weight $k \geq 2$, level $N$, and character $\epsilon$, and,

- $p \mid N$ and the power of $p$ dividing $N$ equals the power of $p$ dividing the conductor of $\epsilon$.

The local Galois representation $\left.\rho_{f}\right|_{G_{p}}: G_{p} \rightarrow \mathrm{GL}_{2}(E)$ attached to $f$ becomes crystalline over a cyclotomic extension $F=\mathbf{Q}_{p}\left(\zeta_{p^{m}}\right)$. Moreover the corresponding filtered module is one of three filtered modules above, as follows.

Let ${ }^{-}$denote complex conjugation. Then the number field $\mathbf{Q}\left(a_{n}\right)$ generated by the Fourier coefficients of $f$ is a CM field, and not a totally real field, since $\bar{a}_{\ell}=a_{\ell} \cdot \epsilon^{-1}(\ell)$ for all primes $\ell \backslash N$ and $\epsilon$ is non-trivial. Decompose $\epsilon=\epsilon_{p} \cdot \epsilon^{\prime}$ into its $p$ and prime-to- $p$ parts, and write $\lambda(a)$ for the unramified character of $W_{p}$ which takes Frob to $a$. Then we claim that the underlying Weil-Deligne representation has trivial monodromy operator (so in particular $\left.\rho_{f}\right|_{G_{p}}: G_{p} \rightarrow \mathrm{GL}_{2}(E)$ is potentially crystalline) and moreover the corresponding representation of $W_{p}$ is given by the sum of characters: $\lambda\left(a_{p}\right) \oplus \epsilon_{p} \cdot \lambda\left(\epsilon^{\prime}(p) \bar{a}_{p}\right)$. This can be seen by noting that the Weil-Deligne representation is known to be an invariant of the compatible system of Galois representations attached to $f$, and so can be computed using a $\lambda$-adic Galois representation attached to $f$, say $V_{\lambda}$, for a place $\lambda$ lying over $\ell \neq p$. Since the local $L$-factor attached to $f$ at $p$ is $1 /\left(1-a_{p} p^{-s}\right)$ we see that the inertial invariants $V_{\lambda}^{I_{p}}$ are one-dimensional and Frob $_{p}$ acts on this line by $a_{p}$, so one character in the Weil representation is $\lambda\left(a_{p}\right)$. To get the other character note that $\operatorname{det}\left(V_{\lambda}\right)$ is $\epsilon \nu_{\ell}^{k-1}=\epsilon_{p} \epsilon^{\prime} \nu_{\ell}^{k-1}$, where $\nu_{\ell}$ is the $\ell$-adic cyclotomic character. Now $\nu_{\ell}$ is unramified at $p$ and takes Frob $_{p}$ to $p$, as is $\epsilon^{\prime}$ which takes $\operatorname{Frob}_{p}$ to $\epsilon^{\prime}(p)$. Moreover by [Miy89, theorem 4.6.17(1)] one has the relation $a_{p} \cdot \bar{a}_{p}=p^{k-1}$. Thus as a character of $W_{p}$ we have $\operatorname{det}\left(V_{\lambda}\right)=\epsilon_{p} \cdot \lambda\left(\epsilon^{\prime}(p) \bar{a}_{p} a_{p}\right)$, so the other character in the Weil representation must be $\epsilon_{p} \cdot \lambda\left(\epsilon^{\prime}(p) \bar{a}_{p}\right)$. Further the type is $\tau=1 \oplus \epsilon_{p}$. Since this is not a scalar the monodromy operator 
must be trivial. Thus $\left.\rho_{f}\right|_{G_{p}}$ is potentially crystalline, and becomes crystalline over $F=\mathbf{Q}_{p}\left(\epsilon_{p}\right)$. This proves the claim.

The discussion above shows that the filtered module associated to $\left.\rho_{f}\right|_{G_{p}}: G_{p} \rightarrow \mathrm{GL}_{2}(E)$ is one of the filtered modules above with $F=\mathbf{Q}_{p}\left(\zeta_{p^{m}}\right)$, where $p^{m}$ is the exact power of $p$ dividing $N$ and $\operatorname{cond}(\epsilon)$, and $\chi_{1}=1$ and $\chi_{2}=\epsilon_{p}$. Moreover the parameters $\alpha$ and $\beta$ in the filtered modules are given by

$$
\alpha=a_{p} \quad \text { and } \quad \beta=\epsilon^{\prime}(p) \bar{a}_{p} .
$$

Now if $f$ is ordinary at $p$ (i.e., $\operatorname{val}_{p}\left(a_{p}\right)=0$ ), then the filtered module associated to $\left.\rho_{f}\right|_{G_{p}}$ is one of $D_{\text {ord-split }}$ or $D_{\text {ord-non-split. }}$. It is known that if $f$ has CM, then the filtered module is $D_{\text {ord-split }}$, and if $f$ does not have CM, then the filtered module is often $D_{\text {ord-non-split (see [GV04], [GV07] for }}$ more precise statements). If on the other hand $f$ is not ordinary at $p$ (i.e., $\operatorname{val}_{p}\left(a_{p}\right)>0$ ), then the associated filtered module is $D_{\text {non-ord }}$.

Finally we note that when

- $p \nmid N$ and so $p$ does not divide the conductor of $\epsilon$,

then we are in the unramified principal series case, and we refer to [Bre01, pp. 30-32] for the corresponding filtered modules (with slightly different normalizations).

3.3. Unramified supercuspidal case. We now assume that the type $\tau$ is as in case (2) of Lemma 2.1, that is, $\left.\tau \simeq \operatorname{Ind}_{W_{p^{2}}}^{W_{p}}(\chi)\right|_{I_{p}}=\left.\left.\chi\right|_{I_{p}} \oplus \chi^{\sigma}\right|_{I_{p}}$, where $\chi$ is a character of $W_{p^{2}}$ which does not extend to $W_{p}$, and is finite on $I_{p}$, and $\sigma$ is the generator of $\operatorname{Gal}\left(\mathbf{Q}_{p^{2}} / \mathbf{Q}_{p}\right)$.

3.3.1. Description of $\operatorname{Gal}\left(F / \mathbf{Q}_{p}\right)$. We must choose a finite extension $F / \mathbf{Q}_{p^{2}}$ over which $\rho$ becomes crystalline, and which simultaneously has the property that $F / \mathbf{Q}_{p}$ is Galois. To do this we use some facts from local class field theory as presented in Iwasawa's book [Iwa86].

Set $K=\mathbf{Q}_{p^{2}}$. For an integer $m \geq 1$, let $K^{m}$ be the unique cyclic unramified extension of $K$ of degree $m$. Fix a uniformizer $\pi$ of $K$ and let $f(X)=\pi X+X^{q}$, where $q=p^{2}$ is the cardinality of the residue field of $K=\mathbf{Q}_{p^{2}}$. Let $W_{f}^{n}$ denote the $(\pi)^{n+1}$-torsion of the $\mathbf{Z}_{p^{2}}$-module whose underlying set is the ring of integers of the completion of the algebraic closure of $K$ and whose $\mathbf{Z}_{p^{2}}$-module structure is induced by the formal group attached to $f(X)$. Then $K\left(W_{f}^{n}\right)$ is a totally ramified abelian extension of $K$, and

$$
\operatorname{Gal}\left(K\left(W_{f}^{n}\right) / K\right) \cong U / U_{n+1}=\mathbf{F}_{q}^{\times} \times \mathbf{Z}_{p^{2}} / \pi^{n},
$$

where $U$ and $U_{n+1}$ are the units, respectively principal units of level $n+1$, of $\mathbf{Z}_{p^{2}}$. Moreover if we choose $\pi \in \mathbf{Q}_{p}$, then $K\left(W_{f}^{n}\right) / \mathbf{Q}_{p}$ is also Galois since $f(X)$ is defined over $\mathbf{Q}_{p}$.

By local class field theory every finite abelian extension $L / K$ is contained in $K^{m} K\left(W_{f}^{n}\right)$, for some $m$ and $n$. Now choose a finite cyclic extension $L / K$ such that $\left.\chi\right|_{I_{L}}=1$. Then $L \subset K^{m} K\left(W_{f}^{n}\right)$ for some $m$ and $n$. We take $F=K^{m} K\left(W_{f}^{n}\right)$, noting that $\left.\tau\right|_{I_{F}}$ is trivial so that $\rho$ is crystalline over $F$. Moreover, with the choice of uniformizer $\pi=p$, which we now fix, the extension $F / \mathbf{Q}_{p}$ is even Galois. 
Let us describe $\operatorname{Gal}\left(F / \mathbf{Q}_{p}\right)$ in more detail. Let $f^{(n)}(X)$ denote the $n$-th iterate of $f(X)$ and let $\alpha$ denote a root of $f^{(n+1)}(X)$ which is not a root of $f^{(n)}(X)$. Then $K\left(W_{f}^{n}\right)=\mathbf{Q}_{p^{2}}(\alpha)$. Now $\mathbf{Q}_{p}(\alpha) / \mathbf{Q}$ is a totally ramified extension of degree equal to the ramification index $e\left(F / \mathbf{Q}_{p}\right)=(q-1) q^{n}$ and $F / \mathbf{Q}_{p}(\alpha)$ is an unramified extension of degree $2 m$. Let $\sigma$ be a generator of $\operatorname{Gal}\left(F / \mathbf{Q}_{p}(\alpha)\right)$. So $\sigma$ has order $2 m$ and projects to the generator of $\operatorname{Gal}\left(K / \mathbf{Q}_{p}\right)$, also denoted $\sigma$ above.

Note $K\left(W_{f}^{0}\right)=\mathbf{Q}_{p^{2}}\left(\pi_{2}\right)$, where $\pi_{2}$ is a root of $X^{p^{2}-1}+p=0$. Set $\Delta:=\operatorname{Gal}\left(K\left(W_{f}^{0}\right) / K\right)$. Then $\Delta$ is isomorphic to the tame part of the inertia subgroup of $\operatorname{Gal}\left(F / \mathbf{Q}_{p}\right)$. Choose a generator $\delta$ of $\Delta$; so $\delta$ has order $p^{2}-1$. Since the order of $\delta$ is prime to $p$, it lifts uniquely to an element, again denoted $\delta$, in $\operatorname{Gal}\left(K\left(W_{f}^{n}\right) / K\right)$, of order $p^{2}-1$ (alternatively use the representation (3.2) of this Galois group as a direct product). This element in turn extends uniquely to an element in $\operatorname{Gal}(F / K) \subset \operatorname{Gal}\left(F / \mathbf{Q}_{p}\right)$ fixing $K^{m}$, and we again denote this element by $\delta$. Finally, let $\Gamma=\mathbf{Z}_{p^{2}} / p^{n}=\mathbf{Z} / p^{n} \oplus \mathbf{Z} / p^{n}$. Then $\Gamma$ is isomorphic to the wild part of the inertia subgroup. Choose generators $\gamma_{1}$ and $\gamma_{2}$ of $\Gamma$; so $\gamma_{1}$, $\gamma_{2}$ each have order $p^{n}$. Again $\gamma_{1}, \gamma_{2}$ lift to elements in $\operatorname{Gal}(F / K) \subset \operatorname{Gal}\left(F / \mathbf{Q}_{p}\right)$ fixing $K^{m}$, and we denote these by the same symbols.

The elements $\delta, \gamma_{1}, \gamma_{2}$ generate the full inertia subgroup $\operatorname{Gal}\left(F / K^{m}\right)$ of $\operatorname{Gal}\left(F / \mathbf{Q}_{p}\right)$. This is a normal subgroup. Since $\sigma^{2}$ fixes $K\left(W_{f}^{n}\right)$ the action of $\sigma$ on $\operatorname{Gal}\left(F / K^{m}\right)$ by conjugation is an involution. This action coincides with the action of $\operatorname{Gal}\left(K / \mathbf{Q}_{p}\right)$ on $\operatorname{Gal}\left(K\left(W_{f}^{n}\right) / K\right)$ by conjugation. Using a standard functorial property of the Artin map [Iwa86, thm. 6.11] this last action is given by the natural action of $\operatorname{Gal}\left(K / \mathbf{Q}_{p}\right)$ on $\mathbf{Z}_{p^{2}}^{\times} / U_{n+1}=\mathbf{F}_{q}^{\times} \times U_{1} / U_{n+1}$ with $U_{1} / U_{n+1} \stackrel{\simeq}{=} \mathbf{Z}_{p^{2}} / p^{n}=$ $\left(\mathbf{Z}_{p} \oplus \mathbf{Z}_{p} \cdot \alpha\right) / p^{n}=\mathbf{Z} / p^{n} \oplus \mathbf{Z} / p^{n} \cdot \bar{\alpha}$, where $\mathbf{Z}_{p^{2}}=\mathbf{Z}_{p}[\alpha]$ and $\alpha^{\sigma}=-\alpha$.

In view of all this, we see that $\operatorname{Gal}\left(F / \mathbf{Q}_{p}\right)$ is a semi-direct product of the cyclic group $\langle\sigma\rangle, \sigma^{2 m}=1$, with the product of three cyclic groups $\langle\delta\rangle \times\left\langle\gamma_{1}\right\rangle \times\left\langle\gamma_{2}\right\rangle, \delta^{p^{2}-1}=1, \gamma_{1}^{p^{n}}=1, \gamma_{2}^{p^{n}}=1$, with the relations

$$
\sigma^{-1} \delta \sigma=\delta^{p}, \quad \sigma^{-1} \gamma_{1} \sigma=\gamma_{1}, \quad \text { and } \quad \sigma^{-1} \gamma_{2} \sigma=\gamma_{2}^{-1} .
$$

We also see that $F_{0}=K^{m}=\mathbf{Q}_{p}^{2 m}$ and that $\operatorname{Gal}\left(F_{0} / \mathbf{Q}_{p}\right)=\langle\sigma\rangle=\mathbf{Z} / 2 m$.

3.3.2. Description of the Galois action. Now recall $D$ is a free $\left(F_{0} \otimes_{\mathbf{Q}_{p}} E\right)$-module of rank 2. Let $D_{i}=D \otimes_{F_{0} \otimes E, \sigma^{i}} E$, for $i=0,1, \ldots, 2 m-1$, be the component of $D$ corresponding to $\sigma^{i}$ thought of as an embedding $F_{0} \hookrightarrow E$. Each $D_{i}$ is a Weil-Deligne representation with an action of $W_{p}$ (the action of $N=0$ ). Moreover by (2.1) the action of $I_{p}$ matches with the action of the inertia subgroup of $\operatorname{Gal}\left(F / \mathbf{Q}_{p}\right)$, namely $\Delta \times \Gamma$. Thinking of $\chi_{\mid I_{p}}$ as a character of this last group, write $\chi=\omega_{2}^{j} \cdot \chi_{1} \cdot \chi_{2}$, where $\omega_{2}$ is the fundamental character of level 2 , and $\chi_{l}$ is the character which takes $\gamma_{l}$ to the $p^{n}$-th root of unity $\zeta_{l}$, for $l=1,2$. Since $\chi$ does not extend to $W_{p}$, we have $\chi \neq \chi^{\sigma}$ on $W_{p^{2}}$, which follows if either $j \not \equiv 0 \bmod p+1$ or $\zeta_{2} \neq \zeta_{2}^{-1}$. In any case we see that each $D_{i}$ has a basis $v_{i 1}, v_{i 2}$ such that, if $i$ is even, then:

$$
\left\{\begin{array}{lll}
\delta \cdot v_{i 1}=\omega_{2}^{j}(\delta) \cdot v_{i 1}, & \gamma_{1} \cdot v_{i 1}=\zeta_{1} \cdot v_{i 1}, & \gamma_{2} \cdot v_{i 1}=\zeta_{2} \cdot v_{i 1} \\
\delta \cdot v_{i 2}=\omega_{2}^{p j}(\delta) \cdot v_{i 2}, & \gamma_{1} \cdot v_{i 2}=\zeta_{1} \cdot v_{i 2}, & \gamma_{2} \cdot v_{i 2}=\zeta_{2}^{-1} \cdot v_{i 2}
\end{array}\right.
$$


and if $i$ is odd, then:

$$
\left\{\begin{array}{lll}
\delta \cdot v_{i 1}=\omega_{2}^{p j}(\delta) \cdot v_{i 1}, & \gamma_{1} \cdot v_{i 1}=\zeta_{1} \cdot v_{i 1}, & \gamma_{2} \cdot v_{i 1}=\zeta_{2}^{-1} \cdot v_{i 1} \\
\delta \cdot v_{i 2}=\omega_{2}^{j}(\delta) \cdot v_{i 2}, & \gamma_{1} \cdot v_{i 2}=\zeta_{1} \cdot v_{i 2}, & \gamma_{2} \cdot v_{i 2}=\zeta_{2} \cdot v_{i 2}
\end{array}\right.
$$

It remains to specify the action of $\sigma$. Using the fact that $\sigma$ takes $D_{i}$ to $D_{i+1}$ and the relations (3.3) we see that:

$$
\begin{gathered}
\sigma \cdot v_{i 1}=a_{i+1} v_{i+1,1}, \\
\sigma \cdot v_{i 2}=b_{i+1} v_{i+1,2},
\end{gathered}
$$

where $a_{i}, b_{i} \in E$ are constants. Applying $\sigma$ repeatedly and noting that $\sigma^{2 m}=1$ we see that $\prod a_{i}=\prod b_{i}=1$. Thus replacing $a_{i+1} v_{i+1,1}$ by $v_{i+1,1}$ (and similarly $b_{i+1} v_{i+1,2}$ by $v_{i+1,2}$ ) we see that we may assume

$$
\begin{gathered}
\sigma \cdot v_{i 1}=v_{i+1,1} \\
\sigma \cdot v_{i 2}=v_{i+1,2}
\end{gathered}
$$

Note that the $v_{i 1}$ are now determined up to multiplication by the same constant (similarly for the $\left.v_{i 2}\right)$.

3.3.3. Definition of action of $\varphi$. The operator $\varphi$ acts in a cyclic manner as well, taking $D_{i}$ to $D_{i+1}$. Since $\varphi$ commutes with, say, the action of the inertia subgroup, we see that:

$$
\begin{aligned}
& \varphi \cdot v_{i 1}=c v_{i+1,2}, \\
& \varphi \cdot v_{i 2}=d v_{i+1,1},
\end{aligned}
$$

where $c$ and $d$ are constants in $E$. The fact that these constants may be taken to be independent of $i$ follows easily from the fact that $\varphi$ commutes with $\sigma$. Now set

$$
\begin{aligned}
& f_{1}=v_{01}+v_{11}+\cdots+v_{2 m-1,1}, \\
& f_{2}=c\left(v_{02}+v_{12}+\cdots+v_{2 m-1,2}\right) .
\end{aligned}
$$

This is a basis for $D$. After what we have said above for the $v_{i 1}$ and the $v_{i 2}$ the vectors $f_{1}$ and $f_{2}$ are fixed up to multiplication by the same constant. Note that $\varphi\left(f_{1}\right)=f_{2}$ and $\varphi\left(f_{2}\right)=\frac{1}{t} f_{1}$, with $\frac{1}{t}=c d \in E$. The admissibility condition implies that $1-k=t_{H}(D)=t_{N}(D)=\operatorname{val}_{p}\left(\frac{1}{t}\right)$ so that $\operatorname{val}_{p}(t)=k-1$.

It will actually be more convenient when computing the filtration in the next subsection to work with an alternative basis of $D$, namely

$$
\begin{aligned}
& e_{1}=\sqrt{d} \cdot v_{01}+\sqrt{c} \cdot v_{12}+\cdots+\sqrt{d} \cdot v_{2 m-2,1}+\sqrt{c} \cdot v_{2 m-1,2}, \\
& e_{2}=\sqrt{c} \cdot v_{02}+\sqrt{d} \cdot v_{11}+\cdots+\sqrt{c} \cdot v_{2 m-2,2}+\sqrt{d} \cdot v_{2 m-1,1}
\end{aligned}
$$

Here we might need to replace $E$ by a finite extension, again denoted by $E$, so that it contains $\sqrt{c}$ and $\sqrt{d}$. Again $e_{1}$ and $e_{2}$ are determined up to multiplication by the same constant, and 
$\varphi\left(e_{1}\right)=\sqrt{c d} \cdot e_{1}$, and $\varphi\left(e_{2}\right)=\sqrt{c d} \cdot e_{2}$. Set $\frac{1}{t}=c d$, as before. The admissibility condition again implies that $1-k=t_{H}(D)=t_{N}(D)=\operatorname{val}_{p}\left(\frac{1}{t}\right)$ so that $\operatorname{val}_{p}(t)=k-1$.

3.3.4. Description of the filtration. There is one last matter to take care of, that of the filtration. For this we mimic some of the ideas used in the ramified principal series case.

Note that every $\operatorname{Gal}\left(F / \mathbf{Q}_{p}\right)$ stable line in $D_{F}$ is spanned by an invariant vector. As in the proof of Lemma 3.1 it suffices to show that $\mathrm{H}^{1}\left(G,\left(F \otimes \mathbf{Q}_{p} E\right)^{\times}\right)=0$, where $G=\operatorname{Gal}\left(F / \mathbf{Q}_{p}\right)$. One could possibly check this directly, as in the proof of Lemma 3.1, but in any case this follows immediately from Remark 3.2.

We now construct a $\operatorname{Gal}\left(F / \mathbf{Q}_{p}\right)$ invariant vector $v=x e_{1}+y e_{2}$, with $x$ and $y \in F \otimes_{\mathbf{Q}_{p}} E$. While it is possible to construct $x$ and $y$ explicitly as in the proof of Proposition 3.3 (this was done in an earlier version of this paper), we follow the referee's suggestion (cf. Remark 3.4) to simplify the construction. First note that $\sigma$ switches $e_{1}$ and $e_{2}$. It follows that $v$ has the form $v=x e_{1}+\sigma(x) e_{2}$ with $\sigma^{2}(x)=x$. Recall that $\chi^{\sigma}$ denotes the character of $\operatorname{Gal}(F / K)$ defined by $\chi^{\sigma}(g)=\chi\left(\sigma^{-1} g \sigma\right)=\chi\left(\sigma g \sigma^{-1}\right)$, where the last equality follows from the fact that $\sigma^{2}$ lies in the abelian group $\operatorname{Gal}(F / K)$. By construction $I(F / K)$ acts on $e_{1}$ and $e_{2}$ by $1 \otimes \chi$ and $1 \otimes \chi^{\sigma}$ respectively. Hence $I(F / K)$ must act on $x$ by $1 \otimes \chi^{-1}$. To construct $x \in F \otimes_{\mathbf{Q}_{p}} E$ with these properties we first note that $\sigma^{2}(x)=x$ forces $x$ to lie in $K\left(W_{f}^{n}\right) \otimes_{\mathbf{Q}_{p}} E$, since $K\left(W_{f}^{n}\right)$ is the fixed field of $\left\langle\sigma^{2}\right\rangle$. Now $K\left(W_{f}^{n}\right) / \mathbf{Q}_{p}$ is a normal extension. By the normal basis theorem choose $\theta \in K\left(W_{f}^{n}\right)$ whose conjugates form a basis of $K\left(W_{f}^{n}\right) / \mathbf{Q}_{p}$. Now every element of $K\left(W_{f}^{n}\right) \otimes \mathbf{Q}_{p} E$, and in particular $x$, can be uniquely expressed as $\sum_{g \in \operatorname{Gal}\left(K\left(W_{f}^{n}\right) / \mathbf{Q}_{p}\right)} g(\theta) \otimes a_{g}$. In view of the structure of $\operatorname{Gal}\left(K\left(W_{f}^{n}\right) / \mathbf{Q}_{p}\right)$ we may further write

$$
x=\sum_{g \in I(F / K)} g(\theta) \otimes a_{g}+\sum_{g \in I(F / K)} \sigma g(\theta) \otimes b_{g} .
$$

Since $I(F / K)$ must act on $x$ by $1 \otimes \chi^{-1}$ we see that $a_{h^{-1} g}=\chi^{-1}(h) a_{g}$ and $b_{h^{-\sigma} g}=\chi^{-1}(h) b_{g}$, for all $h, g \in I(F / K)$, where $h^{-\sigma}:=\sigma^{-1} h^{-1} \sigma$. Taking $g=1$ we see that $x$ has the form:

$$
a \cdot \sum_{g \in I(F / K)} g(\theta) \otimes \chi(g)+b \cdot \sum_{g \in I(F / K)} \sigma g(\theta) \otimes \chi^{\sigma}(g),
$$

where $a, b \in E$. Note that $a$ and $b$ are parameters which determine an element in $\mathbf{P}^{1}(E)$. Now the line

$$
\operatorname{Fil}^{0}\left(D_{F}\right)=\left(F \otimes \mathbf{Q}_{p} E\right)\left(x e_{1}+\sigma(x) e_{2}\right)
$$

is stable by $\operatorname{Gal}\left(F / \mathbf{Q}_{p}\right)$. Since $e_{1}$ and $e_{2}$ are only well determined up to multiplication by the same constant, the isomorphism class of the filtered module is parametrized by $(a, b) \in \mathbf{P}^{1}(E)$.

To summarize, the admissible filtered modules attached to $\rho$ in this case are given by

$$
D_{\text {unr-sc }}[a: b]=\left(F_{0} \otimes_{\mathbf{Q}_{p}} E\right) e_{1} \oplus\left(F_{0} \otimes_{\mathbf{Q}_{p}} E\right) e_{2}
$$


with

$$
D_{\text {unr-sc }}[a: b]=\left\{\begin{array}{rlrl}
\varphi\left(e_{1}\right) & = & \frac{1}{\sqrt{t}} e_{1} \\
\varphi\left(e_{2}\right) & = & \frac{1}{\sqrt{t}} e_{2} \\
t & \in & \mathcal{O}_{E}, & \operatorname{val}_{p}(t)=k-1 \\
N & = & 0 \\
\sigma\left(e_{1}\right) & = & e_{2} \\
\sigma\left(e_{2}\right) & = & & e_{1} \\
g\left(e_{1}\right) & = & (1 \otimes \chi(g)) e_{1}, & g \in I(F / K) \\
g\left(e_{2}\right) & = & \left(1 \otimes \chi^{\sigma}(g)\right) e_{2}, & g \in I(F / K) \\
\operatorname{Fil}^{0}\left(D_{F}\right) & = & \left(F \otimes \mathbf{Q}_{p} E\right)\left(x e_{1}+\sigma(x) e_{2}\right), & x=x(a, b) \\
(a, b) & \in & \mathbf{P}^{1}(E) .
\end{array}\right.
$$

3.4. Ramified supercuspidal case. Let us now assume that $K / \mathbf{Q}_{p}$ is a ramified quadratic extension, and that $\tau=\left.\operatorname{Ind}_{W_{K}}^{W_{p}}(\chi)\right|_{I_{p}}=\operatorname{Ind}_{I_{K}}^{I_{p}}\left(\left.\chi\right|_{I_{K}}\right)$, where $\chi$ is a character of $W_{K}$, finite on $I_{K}$, such that $\left.\chi\right|_{I_{K}}$ does not extend to $I_{p}$. Let $\iota$ be the non-trivial element of $\operatorname{Gal}\left(K / \mathbf{Q}_{p}\right)$.

3.4.1. Description of $\operatorname{Gal}\left(F / \mathbf{Q}_{p}\right)$. As before, we must write down a Galois extension $F$ of $\mathbf{Q}_{p}$ such that $\left.\tau\right|_{I_{F}}=1$. Let $K^{m}$ denote the unramified extension of $K$ of degree $m$, for $m \geq 1$. Let $\pi$ be a uniformizer of $K$, let $f(X)=\pi X+X^{p}$, and as before let $W_{f}^{n}$, for $n \geq-1$, denote the $\left(\pi^{n+1}\right)$ torsion of the ring of integers of the completion of the algebraic closure of $K$, where the $\mathcal{O}_{K}$-module structure is induced by the formal group associated to $f$. Then $K_{\pi}^{n}=K\left(W_{f}^{n}\right)$ is a totally ramified abelian extension of $K$ with

$$
\operatorname{Gal}\left(K_{\pi}^{n} / K\right) \cong U / U_{n+1}=\mathbf{F}_{p}^{\times} \times \mathcal{O}_{K} / \pi^{n}
$$

where $U$ and $U_{n+1}$ are the units, respectively principal units of level $n+1$, of $\mathcal{O}_{K}$. Note that $\mathcal{O}_{K} / \pi^{2 n}=\mathbf{Z} / p^{n} \oplus \mathbf{Z} / p^{n}$. By local class field theory every abelian extension of $K$ is contained in $K^{m} K_{\pi}^{n}$ for some $m, n$.

Now choose $L / K$ finite cyclic such that $\left.\chi\right|_{I_{L}}=1$. Then $L \subset K^{m} K_{\pi}^{n}$ for some $m, n$. Neither $L / \mathbf{Q}_{p}$ nor $K_{\pi}^{n} / \mathbf{Q}_{p}$ is necessarily a Galois extension. The problem of course is that these fields are not preserved by (a lift of) $\iota \in \operatorname{Gal}\left(K / \mathbf{Q}_{p}\right)$. Equivalently, the polynomial $f$ is not defined over $\mathbf{Q}_{p}$ but over $K$. To remedy this assume that $\pi^{\iota}=-\pi$ and consider the Lubin-Tate extension $K_{-\pi}^{n}$ corresponding to the polynomial $f_{-\pi}(X)=-\pi X+X^{p}$. Then $\left(K_{\pi}^{n}\right)^{\iota}=K_{-\pi}^{n}$ and $K_{\pi}^{n} K_{-\pi}^{n}$ is Galois over $\mathbf{Q}_{p}$. Noting $K^{\times}=\langle\pi\rangle \times U_{K}$, we have $\operatorname{Norm}\left(K_{\pi}^{n}\right)=\langle\pi\rangle \times U_{n+1}$ and $\operatorname{Norm}\left(K_{-\pi}^{n}\right)=\langle-\pi\rangle \times U_{n+1}$ so that the intersection of these two norm subgroups is the index two subgroup $\left\langle\pi^{2}\right\rangle \times U_{n+1}$. Since the composition of abelian extensions of $K$ corresponds (via local class field theory) to the intersection of the corresponding norm subgroups we see that $K_{\pi}^{n} K_{-\pi}^{n}$ is an index two over-field of $K_{\pi}^{n}$ obtained by composing $K_{\pi}^{n}$ with an unramified quadratic extension. Indeed if $K_{\pi}^{n}=K(\alpha)$, then $K_{-\pi}^{n}=K(\beta)$ with $\beta=\zeta \alpha$ and $\zeta^{p-1}=-1$, and $K_{\pi}^{n} K_{-\pi}^{n}=K(\alpha, \zeta)$. Replacing $m$ by $2 m$ to ensure it is even we see that $K^{2 m} K_{\pi}^{n}$ is a Galois extension of $\mathbf{Q}_{p}$. Finally since $\mathcal{O}_{K} / \pi^{2 n}=\mathbf{Z} / p^{n} \oplus \mathbf{Z} / p^{n}$, we will find it convenient to replace $n$ by $2 n$ so that it too is even. 
Now set $F=K^{2 m} K_{\pi}^{2 n}$. From what we have just said $F / \mathbf{Q}_{p}$ is a Galois extension containing both $L$ and $L^{\iota}$. In particular $\left.\tau\right|_{I_{F}}=1$ and $\rho$ becomes crystalline over $F$.

In order to describe the Galois group of $F / \mathbf{Q}_{p}$ explicitly, note that there is an exact sequence

$$
1 \rightarrow \operatorname{Gal}(F / K) \rightarrow \operatorname{Gal}\left(F / \mathbf{Q}_{p}\right) \rightarrow \operatorname{Gal}\left(K / \mathbf{Q}_{p}\right) \rightarrow 1
$$

where

$$
\operatorname{Gal}(F / K)=\operatorname{Gal}\left(F / K_{\pi}^{2 n}\right) \times \operatorname{Gal}\left(F / K^{2 m}\right)=\langle\sigma\rangle \times(\Delta \times \Gamma), \quad \operatorname{Gal}\left(K / \mathbf{Q}_{p}\right)=\langle\iota\rangle,
$$

with $\sigma^{2 m}=1$, and

$$
\begin{aligned}
\Delta & =\mathbf{F}_{p}^{\times}=\langle\delta\rangle \text { with } \delta^{p-1}=1 \\
\Gamma & =\mathcal{O}_{K} / \pi^{2 n}=\left\langle\gamma_{1}\right\rangle \times\left\langle\gamma_{2}\right\rangle \text { with } \gamma_{i}^{p^{n}}=1 \text { for } i=1,2 .
\end{aligned}
$$

Here $\Delta$ and $\Gamma$ are the tame and wild parts of the inertia subgroup $I(F / K)=\operatorname{Gal}\left(F / K^{2 m}\right)$.

We now claim that the exact sequence (3.4) does not split. Write $\iota \in I\left(F / \mathbf{Q}_{p}\right)$ for a lift of the order 2 element $\iota \in I\left(K / \mathbf{Q}_{p}\right)=\operatorname{Gal}\left(K / \mathbf{Q}_{p}\right)$. Then this element does not have order 2 in $\operatorname{Gal}\left(F / \mathbf{Q}_{p}\right)$, since otherwise it would have order prime to $p$ and would lie in the tame part of $I\left(F / \mathbf{Q}_{p}\right)$. But this subgroup already contains the order 2 element $\delta^{(p-1) / 2}$ which is necessarily different from $\iota$ since it fixes $K$. This means that the tame part of $I\left(F / \mathbf{Q}_{p}\right)$ is not cyclic, a contradiction.

However we may assume that $\iota^{2}=\delta$. Indeed note that $I(F / K)$ is a normal subgroup of $I\left(F / \mathbf{Q}_{p}\right)$ and the conjugation action of $\iota$ is given by:

$$
s \iota^{-1} \delta \iota=\delta, \quad \iota^{-1} \gamma_{1} \iota=\gamma_{1}, \quad \text { and } \quad \iota^{-1} \gamma_{2} \iota=\gamma_{2}^{-1}
$$

These relations can be checked using the functoriality of the Artin map as in the unramified supercuspidal case. Thus the action of $\iota$ on $\delta, \gamma_{1}$ and $\gamma_{2}$ can be determined by looking at the action of $\iota$ on $K^{\times} / \operatorname{Norm}\left(K_{\pi}^{2 n}\right)=\mathbf{F}_{p}^{\times} \times U_{1} / U_{2 n+1}$, with $U_{1} / U_{2 n+1} \cong \mathcal{O}_{K} /(\pi)^{2 n}=\left(\mathbf{Z}_{p} \oplus \mathbf{Z}_{p} \cdot \pi\right) /(p)^{n}=$ $\mathbf{Z} / p^{n} \oplus \mathbf{Z} / p^{n} \cdot \bar{\pi}$, and noting $\pi^{\iota}=-\pi$. In any case the relations (3.5) along with the identity $\left(\iota^{2}\right) \iota=\iota\left(\iota^{2}\right)$ show that $\iota^{2}$ belongs to the center of $I\left(F / \mathbf{Q}_{p}\right)$, and hence $\iota^{2}=\delta^{a} \gamma_{1}^{b}$ for some $a, b$. Since $b \equiv 2 c \bmod p^{n}$ for some $c$, we may modify $\iota$ to assume $b=0$. We may similarly modify $\iota$ so that $a=0$ or 1 , but after what has been said above about the cyclicity of tame inertia, we must have $a=1$. Thus we may assume that $\iota^{2}=\delta$, as claimed.

It remains to describe the commutator of $\iota$ and $\sigma$. Note that $\iota \sigma \iota^{-1} \neq \sigma$ since as remarked above $K_{\pi}^{2 n} / \mathbf{Q}_{p}$ is not a normal extension. However $L=K_{\pi}^{2 n} K_{-\pi}^{2 n}=K(\alpha, \zeta)$, the fixed field of $\left\langle\sigma^{2}\right\rangle$, is a normal extension of $\mathbf{Q}_{p}$, and an easy check using the Artin map once more shows that $\iota \sigma^{2} \iota^{-1}=\sigma^{2}$. Indeed $L^{\times} / \operatorname{Norm}(F)=\left(\langle\alpha\rangle \times \mathcal{O}_{L}^{\times}\right) /\left(\left\langle\alpha^{m}\right\rangle \times \mathcal{O}_{L}^{\times}\right)$and $\alpha^{\iota}=\zeta \alpha$ and $\zeta$ is a norm. It follows that $\iota \sigma \iota^{-1} \sigma^{-1}$ is an order 2 element of $\operatorname{Gal}(F / K)$, and so must be either $\sigma^{m}$, or $\delta^{(p-1) / 2}$, or a product of these two elements. The first and last cases are impossible since $\iota \sigma \iota^{-1} \sigma^{-1}$ fixes $\mathbf{Q}_{p^{2 m}}$, so in particular fixes the group of $\left(p^{2 m}-1\right)$-th roots of 1 , whereas $\sigma^{m}: u \mapsto u^{p^{m}}$ acts non-trivially on this group. Hence

$$
\iota^{-1} \sigma \iota=\sigma \cdot \delta^{(p-1) / 2}
$$


Summarizing we see that $\operatorname{Gal}\left(F / \mathbf{Q}_{p}\right)$ is generated by the abelian index two subgroup $\operatorname{Gal}(F / K)=$ $\langle\sigma\rangle \times \Delta \times \Gamma$, with notation as above, and the element $\iota$ satisfying $\iota^{2}=\delta \in \Delta$ and the commutation relations (3.5), (3.6) above. We note that $F_{0}=\mathbf{Q}_{p^{2 m}}$ and the inertia subgroup of $\operatorname{Gal}\left(F / \mathbf{Q}_{p}\right)$ is a (non-split) extension of $\Delta \times \Gamma$ by a group of order 2 .

3.4.2. Description of the Galois action. Now $D$ is a free $\left(F_{0} \otimes_{\mathbf{Q}_{p}} E\right)$-module of rank 2. As before set $D_{i}=D \otimes_{F_{0} \otimes E, \sigma^{i}} E$, for $i=0,1, \ldots, 2 m-1$. Each $D_{i}$ is a Weil-Deligne representation and the action of $I_{p}$ is given by the action of the inertia subgroup of $\operatorname{Gal}\left(F / \mathbf{Q}_{p}\right)$. Now write $\left.\chi\right|_{I_{K}}=\omega^{j} \cdot \chi_{1} \cdot \chi_{2}$, where $\omega$ is the Teichmüller character, and $\chi_{i}\left(\gamma_{i}\right)=\zeta_{i}$, with $\zeta_{i}$ a $p^{n}$-th root of 1 , for $i=1,2$ (the condition that $\left.\chi\right|_{I_{K}}$ does not extend to $I_{p}$ is equivalent to $\left.\zeta_{2} \neq \zeta_{2}^{-1}\right)$. It follows that there is a basis $v_{i 1}, v_{i 2}$ of $D_{i}$ such that:

$$
\left\{\begin{array} { l } 
{ \iota \cdot v _ { i 1 } = v _ { i 2 } } \\
{ \iota \cdot v _ { i 2 } = \omega ^ { j } ( \delta ) \cdot v _ { i 1 } }
\end{array} \quad \text { and } \quad \left\{\begin{array}{lll}
\delta \cdot v_{i 1}=\omega^{j}(\delta) \cdot v_{i 1}, & \gamma_{1} \cdot v_{i 1}=\zeta_{1} \cdot v_{i 1}, & \gamma_{2} \cdot v_{i 1}=\zeta_{2} \cdot v_{i 1}, \\
\delta \cdot v_{i 2}=\omega^{j}(\delta) \cdot v_{i 2}, & \gamma_{1} \cdot v_{i 2}=\zeta_{1} \cdot v_{i 2}, & \gamma_{2} \cdot v_{i 2}=\zeta_{2}^{-1} \cdot v_{i 2} .
\end{array}\right.\right.
$$

Note that for each $i$, the vectors $v_{i 1}$ and $v_{i 2}$ are determined up to multiplication by the same constant.

Since $\sigma$ commutes with $\gamma_{2}$ and $\sigma: D_{i} \rightarrow D_{i+1}$, arguments similar to those in section 3.3.2, along with the relation (3.6), show that we may assume

$$
\begin{aligned}
& \sigma \cdot v_{i 1}=v_{i+1,1}, \\
& \sigma \cdot v_{i 2}=(-1)^{j} \cdot v_{i+1,2} .
\end{aligned}
$$

We note that $\omega^{j}\left(\delta^{(p-1) / 2}\right)=(-1)^{j}$. Now all the $v_{i 1}$ and $v_{i 2}$ are determined up to multiplication by the same constant (independent of $i$ ).

3.4.3. Definition of action of $\varphi$. Since $\varphi: D_{i} \rightarrow D_{i+1}$ commutes with the action of $\operatorname{Gal}\left(F / \mathbf{Q}_{p}\right)$ and in particular with the action of $\gamma_{2}$ we see that

$$
\begin{aligned}
& \varphi \cdot v_{i 1}=c v_{i+1,1}, \\
& \varphi \cdot v_{i 2}=d v_{i+1,2},
\end{aligned}
$$

where $c$ and $d$ are constants. Again the fact that these constants can be taken independent of $i$ follows from the fact that $\varphi$ commutes with $\sigma$. Moreover, since $\varphi$ commutes with $\iota$, we must have $c=d$. Set

$$
\begin{aligned}
& e_{1}=v_{01}+v_{11}+\cdots+v_{2 m-1,1}, \\
& e_{2}=v_{02}+v_{12}+\cdots+v_{2 m-1,2} .
\end{aligned}
$$

After what has been said above concerning the $v_{i j}$ we see that $e_{1}$ and $e_{2}$ are determined up to multiplication by the same constant. Clearly $\varphi\left(e_{1}\right)=c e_{1}$ and $\varphi\left(e_{2}\right)=c e_{2}$. Set $t=1 / c^{2}$. The admissibility condition implies that $\operatorname{val}_{p}(t)=k-1$. 
3.4.4. Definition of the filtration. Finally we describe the filtration on $D_{F}$. As in section 3.3.4 every $\operatorname{Gal}\left(F / \mathbf{Q}_{p}\right)$ stable line in $D_{F}$ is spanned by an invariant vector. Say $v=x e_{1}+y e_{2}$ is such a vector. Note that $I(F / K)$ acts on $e_{1}$ and $e_{2}$ by $\chi$ and $\chi^{\iota}$ respectively, where $\chi^{\iota}(g)=\chi\left(\iota^{-1} g \iota\right)$, for $g \in \operatorname{Gal}(F / K)$. Thus $I(F / K)$ must act on $x$ and $y$ by $1 \otimes \chi^{-1}$ and $1 \otimes\left(\chi^{\iota}\right)^{-1}$ respectively. Since $\iota e_{1}=e_{2}$ and $\iota e_{2}=\left(1 \otimes \omega^{j}(\delta)\right) e_{1}$, we see that $v$ has the form $x e_{1}+\iota(x) e_{2}$. Further since $\sigma$ fixes $e_{1}$ and acts by $(-1)^{j}$ on $e_{2}$ and satisfies the commutation relation (3.6), we see that $\sigma(v)=v$ forces $\sigma(x)=x$.

To construct $x \in F \otimes_{\mathbf{Q}_{p}} E$ with these properties we proceed as before. First note that $\sigma(x)=x$ implies that $x \in K_{\pi}^{2 n} \otimes \mathbf{Q}_{p} E$. Choose $\theta \in K_{\pi}^{2 n}$ such that the conjugates of $\theta$ under the action of $I(F / K) \cong \operatorname{Gal}\left(K_{\pi}^{2 n} / K\right)$ form a basis of $K_{\pi}^{2 n}$ over $K=\mathbf{Q}_{p}(\pi)$. Then the elements $g(\theta), \pi g(\theta)$, for $g \in I(F / K)$ form a basis of $K_{\pi}^{2 n}$ over $\mathbf{Q}_{p}$. Now every element in $K_{\pi}^{2 n} \otimes_{\mathbf{Q}_{p}} E$, and $x$ in particular, can be written uniquely as

$$
\sum_{g \in I(F / K)} g(\theta) \otimes a_{g}+\sum_{g \in I(F / K)} \pi g(\theta) \otimes b_{g} .
$$

Using the action of $I(F / K)$ we see that $a_{h^{-1} g}=\chi^{-1}(h) a_{g}$ for all $h, g \in \operatorname{Gal}(F / K)$, and similarly for $b_{g}$. Taking $g=1$ we see that $x$ has the form:

$$
a \cdot \sum g(\theta) \otimes \chi(g)+b \cdot \sum \pi g(\theta) \otimes \chi(g),
$$

where $a, b \in E$. Then $\operatorname{Fil}^{0}\left(D_{F}\right)=\left(F \otimes_{\mathbf{Q}_{p}} E\right)\left(x e_{1}+\iota(x) e_{2}\right)$ for some $a, b \in E$. Since $e_{1}$ and $e_{2}$ are only determined up to multiplication by the same constant, $(a, b) \in \mathbf{P}^{1}(E)$ parametrize all possibilities for $\operatorname{Fil}^{0}\left(D_{F}\right)$.

To summarize, the admissible filtered modules attached to $\rho$ are given by

$$
D_{\text {ram-sc }}[a: b]=\left(F_{0} \otimes \mathbf{Q}_{p} E\right) e_{1} \oplus\left(F_{0} \otimes_{\mathbf{Q}_{p}} E\right) e_{2}
$$

with

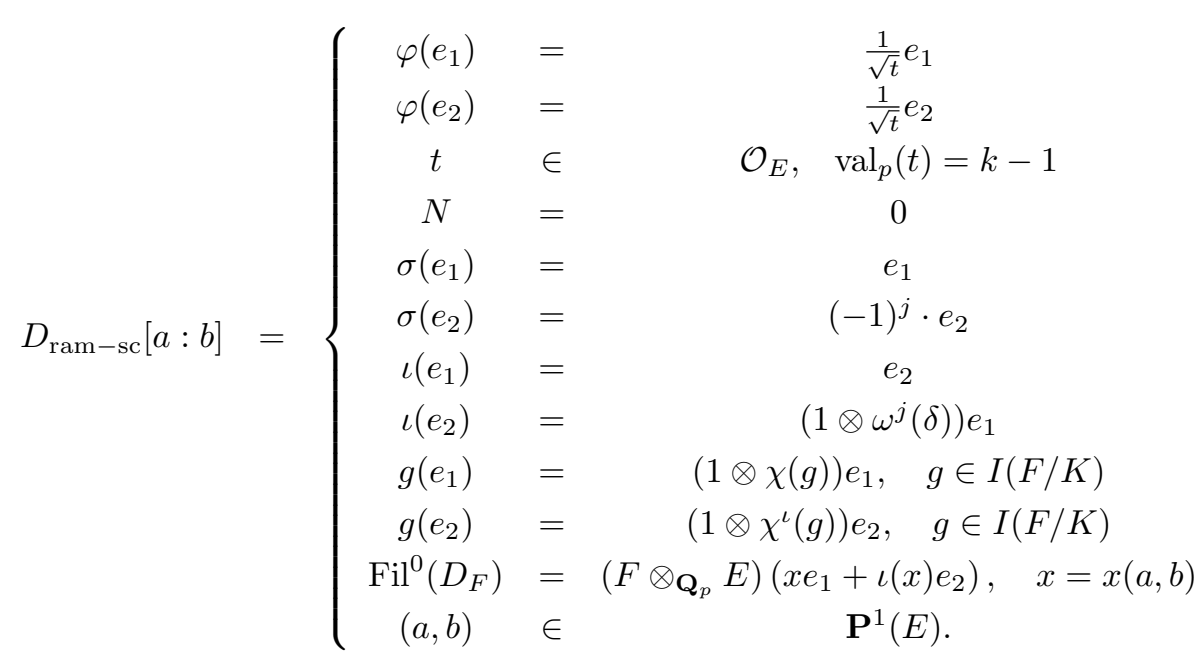


In both the unramified and ramified supercuspidal cases the filtration depends on a parameter in $\mathbf{P}^{1}(E)$. When $f$ is a primitive elliptic modular cusp form of weight $k \geq 2$, level $N$, and character $\epsilon$, and $p^{2} \mid N$ but the power of $p$ dividing $N$ is different from the power of $p \operatorname{dividing} \operatorname{cond}(\epsilon)$, then the associated filtered module can be of supercuspidal type. In such cases it seems to be an open and intriguing question to relate this parameter to the underlying form.

Acknowledgements. The authors thank C. Breuil for suggesting that we work on this problem. We also wish to thank the referee for a very careful reading of the paper, for correcting an error in the last section, and for simplifying some of our arguments.

\section{REFERENCES}

[Ber04] L. Berger. An introduction to the theory of p-adic representations, In Geometric aspects of Dwork theory, Vols. I, II, Walter de Gruyter GmbH 8 Co. KG, Berlin (2004), 255-292.

[Ber05] L. Berger. Représentations modulaires de $\mathrm{GL}_{2}\left(\mathbf{Q}_{p}\right)$ et représentations galoisiennes de dimension 2 , To appear in Astérisque (2005).

[BB06] L Berger and C. Breuil. Sur quelques représentations potentiellement cristallines de $\mathrm{GL}_{2}\left(\mathbf{Q}_{p}\right)$, To appear in Astérisque (2006).

[Bre01] C. Breuil. Lectures on p-adic Hodge theory, deformations and local Langlands, Volume 20, Advanced course lecture notes. Centre de Recerca Matemàtica, Barcelona (2001).

[Bre03] C. Breuil. Sur quelques représentations modulaires et p-adiques de $\mathrm{GL}_{2}\left(\mathbf{Q}_{p}\right)$ II, J. Inst. Math. Jussieu, 2 (2003), 23-58.

[Bre04] C. Breuil. Invariant $\mathfrak{L}$ et série spéciale p-adique, Ann. Scient. de l'ENS, 37 (2004), 559-610.

[BCDT] C. Breuil, B. Conrad, F. Diamond and R. Taylor. On the modularity of elliptic curves over Q: wild 3-adic exercices, J. Amer. Soc, 14 (2001), 843-939.

[BE05] C. Breuil and M. Emerton. Représentations p-adiques ordinaires de $\mathrm{GL}_{2}\left(\mathbf{Q}_{p}\right)$ et compatibilité local-global, To appear in Astérisque (2005).

[BM02] C. Breuil and A. Mézard. Multiplicités modulaires et représentations de $\mathrm{GL}_{2}\left(\mathbf{Z}_{p}\right)$ et de $\operatorname{Gal}\left(\overline{\mathbf{Q}}_{p} / \mathbf{Q}_{p}\right)$ en $l=p$, Duke Math. J., 115 (2002), 205-310.

[BM05] C. Breuil and A. Mézard. Représentations semi-stables de $\mathrm{GL}_{2}\left(\mathbf{Q}_{p}\right)$, demi-plan p-adique et réduction modulo p, To appear in Astérisque (2005).

[Col04] P. Colmez. Une correspondance de Langlands locale p-adique pour les représentations semi-stables de dimension 2, To appear in Astérisque (2004).

[Col05] P. Colmez. Série principale unitaire pour $\mathrm{GL}_{2}\left(\mathbf{Q}_{p}\right)$ et représentations triangulines de dimension 2 , To appear in Astérisque (2005).

[Col07] P. Colmez. La série principale unitaire de $\mathrm{GL}_{2}\left(\mathbf{Q}_{p}\right)$, To appear in Astérisque (2007).

[CF00] P. Colmez and J.-M. Fontaine. Construction des représentations p-adiques semi-stables, Invent. Math., 140 (2000), 1-43.

[Eme05] M. Emerton. p-adic L-functions and unitary completions of representations of p-adic reductive groups, Duke Math. J, 130 (2005), 353-392.

[Fon94a] J.-M. Fontaine. Représentations p-adiques semi-stables, Astérisque, 223, Soc. Math. de France (1994), 113-184.

[Fon94b] J.-M. Fontaine. Le corps des périodes p-adiques, Astérisque, 223, Soc. Math. de France (1994), 59-111.

[FM95] J.-M. Fontaine and B. Mazur. Geometric Galois representations. In Elliptic curves, modular forms, \& Fermat's last theorem. Proceedings of the conference on elliptic curves and modular forms held at the 
Chinese University of Hong Kong, December 18-21, 1993. International Press. Ser. Number Theory. 1, 41-78 (1995)

[GV04] E. Ghate and V. Vatsal. On the local behaviour of ordinary $\Lambda$-adic representations, Ann. Inst. Fourier (Grenoble), 54 (2004), 2143-2162.

[GV07] E. Ghate and V. Vatsal. Locally indecomposable Galois representations, To appear in Canad. J. Math. (2007).

[Iwa86] K. Iwasawa. Local class field theory, volume 1400 of Oxford Science Publications. Oxford Mathematical Monographs. The Clarendon Press, Oxford University Press, New York, 1986.

[Maz94] B. Mazur. On monodromy invariants occurring in global arithmetic, and Fontaine's theory. In p-adic monodromy and the Birch and Swinnerton-Dyer conjecture (Boston, MA, 1991), volume 165 of Contemp. Math., pages 1-20. Amer. Math. Soc., Providence, RI, 1994.

[Miy89] T. Miyake. Modular Forms. Springer-Verlag, 1989.

[Sav05] D. Savitt. On a conjecture of Conrad, Diamond and Taylor, Duke Math. J., 128 (2005), 141-197.

[Vol01] M. Volkov. Les représentations $\ell$-adiques associées aux courbes elliptiques sur $\mathbb{Q}_{p}$. J. Reine Angew. Math., 535:65-101, 2001.

School of Mathematics, Tata Institute of Fundamental Research, Homi Bhabha Road, Mumbai 400005, INDIA.

E-mail address: eghate@math.tifr.res.in

Laboratoire de mathématiques, Faculté des sciences d’Orsay, Université de Paris-Sud 11, 91405 Orsay Cedex, France. Current address: laboratoire de Mathématiques de Versailles, Université de Versailles Saint-Quentin, 45 avenue des États Unis, 78035 Versailles, France.

E-mail address: mezard@math.uvsq.fr 